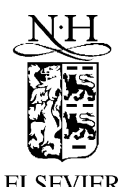

Nuclear Physics B 596 [FS] (2001) 525-547

www.elsevier.nl/locate/npe

\title{
Exact solution for the Bariev model with boundary fields
}

\author{
A. Foerster ${ }^{\text {a }}$, X.-W. Guan ${ }^{\text {a,* }}$, J. Links ${ }^{\text {c }}$, I. Roditi ${ }^{\text {b }}$, H.-Q. Zhou ${ }^{\text {c }}$

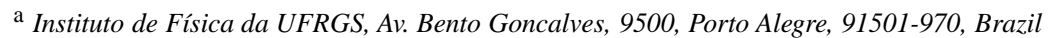 \\ ${ }^{\mathrm{b}}$ Centro Brasileiro de Pesquisas Físicas, Rua Dr. Xavier Sigaud 150, 22290-180, Rio de Janeiro- RJ, Brazil \\ c Centre for Mathematical Physics, Department of Mathematics, The University of Queensland, 4072, Australia
}

Received 6 September 2000; accepted 16 November 2000

\begin{abstract}
The Bariev model with open boundary conditions is introduced and analysed in detail in the framework of the Quantum Inverse Scattering Method. Two classes of independent boundary reflecting $K$-matrices leading to four different types of boundary fields are obtained by solving the reflection equations. The models are exactly solved by means of the algebraic nested Bethe ansatz method and the four sets of Bethe ansatz equations as well as their corresponding energy expressions are derived. @ 2001 Elsevier Science B.V. All rights reserved.
\end{abstract}

PACS: 71.10.-w; 71.10.Fd; 75.10.Jm

Keywords: Integrable spin chains; Algebraic Bethe ansatz; Yang-Baxter algebra; Reflection equations

\section{Introduction}

Much work has been devoted, during the last years, towards a better understanding of integrable models of strongly correlated electrons. Such an interest arises from the efforts to unveil a theory that can provide a consistent picture for observed phenomena of condensed matter physics such as high- $T_{c}$ superconductivity. Two prototypes of integrable models for this purpose are the one-dimensional (1D) Hubbard model solved by Lieb and $\mathrm{Wu}$ [1] (see also [2]) and the $t-J$ model [3] (which is integrable for the supersymmetric coupling [4]), mainly because of their relevance in the description of electronic mechanisms. This is justified on physical grounds since the electron hopping is strongly disturbed by the on-site Coulomb interaction in the 1D Hubbard model and by the spin fluctuations through the antiferromagnetic coupling for the supersymmetric

\footnotetext{
* Corresponding author.

E-mail address: guan@if.ufrgs.br (X.-W. Guan).
} 
$t-J$ model. More recently, motivated by the inclusion of additional interactions whether through internal impurities or external boundary fields, a renewed interest in the study of integrable strongly correlated electron systems has taken place. Some examples of the latter are the 1D Hubbard open chain [5], the supersymmetric $t-J$ model with boundary fields $[6,7]$ and other integrable electronic systems with boundary interactions associated with Lie superalgebra symmetries [8].

The study of integrable systems with open boundary conditions (OBC) [5,9-13] has turned into an active domain since the introduction by Sklyanin [14] of a generalization of the Quantum Inverse Scattering Method (QISM) [15,16] which provides a systematic approach to handle the boundary problem by introducing a set of equations, called reflection equations (RE). The solutions of these equations, referred to as the boundary $K$-matrices, in turn introduce boundary interactions into the Hamiltonian of the system in such a manner that integrability is preserved. Indeed, the presence of boundary fields drastically changes surface critical properties, and in some occasions results in the appearance of so-called boundary bound states [17-19]. Nevertheless, in spite of these results, several models still lack a comprehensive study regarding $\mathrm{OBC}$, one of those being the 1D Bariev model [20,21].

The 1D Bariev (interacting $X Y$ ) chain is also a Hubbard-like integrable model of special interest, as it exhibits the existence of hole pairs of Cooper type which are relevant to theories of superconductors. The integrability of the 1D Bariev model within the framework of the QISM was first established by Zhou in [22] (see also [23]). To achieve this goal one has to build non-trivial higher conserved currents that allow the identification of a quantum $R$-matrix satisfying the Yang-Baxter equation $[15,16]$. This $R$-matrix does not possess the difference property for the spectral parameter, a feature that is also shared by the $R$-matrix associated with the Hubbard model [24]. Soon after this parametrization of the model had been obtained, the algebraic Bethe ansatz solution for the periodic chain was calculated independently by Martins and Ramos [25] and by Zhou [26]. On the other hand, as far as we know, studies concerning OBC for the Bariev model appear incomplete. This can be understood in light of the fact that the $R$-matrix is presented in a complicated form with the absence of both additivity for the spectral parameter and crossing unitarity. As such, we must resort to searching for solutions of a much more intricate RE. Though some known solutions to the RE for the 1D Bariev open chain provide a certain class of boundary $K$-matrices [27], a thorough investigation is still lacking and, moreover, the algebraic Bethe ansatz solution has not been obtained yet.

In this paper, we shall study in detail the OBC for the 1D Bariev chain within the framework of the QISM. We will show that besides the $K$-matrices obtained in [27] there exists a new class of solutions of the RE which leads to pure magnetic boundary fields in the Hamiltonian and provides more general nesting boundary $K$-matrices for a hidden asymmetric $X X Z$ open chain. Here it is worth noticing that this class of boundary fields may have a feasible realization by applying boundary external voltages in experiments on quantum wires [28]. By means of the algebraic Bethe ansatz technique, we obtain the eigenvalues of the transfer matrix of the 1D Bariev model for four different kinds of possible boundary fields. For all cases we present the Bethe ansatz equations and the 
energy spectrum in explicit form, which provides a first step towards investigating the surface critical properties for the 1D Bariev model with these different boundary fields.

We mention finally that we are only concerned here with diagonal solutions of the reflection equations. The case of non-diagonal solutions remains an open problem to be investigated at a later date. There is a general belief that the number of diagonal solutions to each reflection equation is equal to the rank of the symmetry algebra which underlies the solution of the Yang-Baxter equation. This is true, for example, in the case of the $A_{n}$ series of solutions [11] where there are $n$ solutions for each reflection equation, and for the integrable $t-J$ chain where there are 2 solutions which is the rank of the symmetry algebra $U_{q}(\operatorname{sl}(2 \mid 1))$ [12]. However, we are unaware of any proof of this statement in general. For the Bariev model, the symmetry of the $R$-matrix is given by $U_{q}(s l(1 \mid 1)) \times U_{q}(s l(1 \mid 1))$ which has rank 2 . We therefore expect that our results provide the full set of diagonal reflection equation solutions for this model.

The paper is organized as follows. In Section 2 we construct the Bariev model with four kinds of boundary fields by means of the QISM adapted to special boundary conditions. The basic quantities, e.g., the $R$-matrix, the matrices $K_{ \pm}$defining the boundaries, the monodromy matrices and the transfer matrices are defined. In particular, we solve the RE and obtain two independent classes of solutions for the boundary $K$-matrices using the variable-separation method [29]. The relation between the transfer matrices and the Bariev Hamiltonians with different boundary fields is established. Sections 3 and 4 are devoted to the solution of the models through Bethe ansatz methods. In particular, in Section 3 we discuss the action of the transfer matrix on the pseudovacuum state. In Section 4 we perform the nested algebraic Bethe ansatz and find the Bethe ansatz equations, the eigenvalues of the transfer matrices and the energy spectrum of the system. Section 5 is reserved for our conclusions and discussions.

\section{The Bariev open chain with boundary fields}

Let us begin by introducing the Bariev model with boundary fields whose Hamiltonian reads

$$
\begin{aligned}
H=\sum_{j=1}^{N-1}\{ & \left\{\sigma_{j}^{+} \sigma_{j+1}^{-}+\sigma_{j}^{-} \sigma_{j+1}^{+}\right) \exp \left(\beta \tau_{j+1}^{+} \tau_{j+1}^{-}\right) \\
& \left.+\left(\tau_{j}^{+} \tau_{j+1}^{-}+\tau_{j}^{-} \tau_{j+1}^{+}\right) \exp \left(\beta \sigma_{j}^{+} \sigma_{j}^{-}\right)\right\}+ \text {b.t., }
\end{aligned}
$$

where the four different boundary terms are given by

$$
\begin{aligned}
\text { b.t. }{ }^{(\mathrm{I}, \mathrm{I})}= & -\frac{\exp (\beta)}{c_{2}}\left[\sinh \beta \sigma_{N}^{z} \tau_{N}^{z}+\cosh \beta\left(\sigma_{N}^{z}+\tau_{N}^{z}\right)\right] \\
& -\frac{\exp (\beta)}{c_{1}}\left[\sinh \beta \sigma_{1}^{z} \tau_{1}^{z}+\cosh \beta\left(\sigma_{1}^{z}+\tau_{1}^{z}\right)\right], \\
\text { b.t. }{ }^{(\mathrm{II}, \mathrm{II})}= & \frac{1}{2} \exp (\beta) c_{2} \sigma_{N}^{z}+\frac{1}{2} \exp (\beta) c_{1} \tau_{1}^{z},
\end{aligned}
$$




$$
\begin{aligned}
\text { b.t. }{ }^{(\mathrm{I}, \mathrm{II})}= & -\frac{\exp (\beta)}{c_{2}}\left[\sinh \beta \sigma_{N}^{z} \tau_{N}^{z}+\cosh \beta\left(\sigma_{N}^{z}+\tau_{N}^{z}\right)\right] \\
& +\frac{1}{2} \exp (\beta) c_{1} \tau_{1}^{z}, \\
\text { b.t. }^{(\mathrm{II}, \mathrm{I})}= & \frac{1}{2} \exp (\beta) c_{2} \sigma_{N}^{z} \\
& -\frac{\exp (\beta)}{c_{1}}\left[\sinh \beta \sigma_{1}^{z} \tau_{1}^{z}+\cosh \beta\left(\sigma_{1}^{z}+\tau_{1}^{z}\right)\right] .
\end{aligned}
$$

Above $\sigma_{i}$ and $\tau_{i}$ are the two commuting species of Pauli matrices acting on site $i, \beta$ is a coupling constant and $c_{1}, c_{2}$ are the boundary parameters characterizing the strength of the boundary fields. Notice that in cases (2.2), (2.3) the boundary terms at the left and right ends are consistent with the bulk symmetry which is a combination of the inversion $j \rightarrow N-j+1$ and the exchange $\sigma \leftrightarrow \tau$. The cases (2.4), (2.5) are interchanged under this symmetry.

In order to derive these models, we first consider the following $R$-matrix [22]

$R(u, v)$

$$
=\left(\begin{array}{cccccccccccccccc}
\rho_{1} & 0 & 0 & 0 & 0 & 0 & 0 & 0 & 0 & 0 & 0 & 0 & 0 & 0 & 0 & 0 \\
0 & \rho_{2} & 0 & 0 & \rho_{3} & 0 & 0 & 0 & 0 & 0 & 0 & 0 & 0 & 0 & 0 & 0 \\
0 & 0 & \rho_{2} & 0 & 0 & 0 & 0 & 0 & \rho_{3} & 0 & 0 & 0 & 0 & 0 & 0 & 0 \\
0 & 0 & 0 & \rho_{4} & 0 & 0 & \rho_{5} & 0 & 0 & \rho_{6} & 0 & 0 & \rho_{9} & 0 & 0 & 0 \\
0 & \rho_{3} & 0 & 0 & \rho_{2} & 0 & 0 & 0 & 0 & 0 & 0 & 0 & 0 & 0 & 0 & 0 \\
0 & 0 & 0 & 0 & 0 & \rho_{1} & 0 & 0 & 0 & 0 & 0 & 0 & 0 & 0 & 0 & 0 \\
0 & 0 & 0 & \rho_{12} & 0 & 0 & \rho_{7} & 0 & 0 & \rho_{15} & 0 & 0 & \rho_{5} & 0 & 0 & 0 \\
0 & 0 & 0 & 0 & 0 & 0 & 0 & \rho_{9} & 0 & 0 & 0 & 0 & 0 & \rho_{11} & 0 & 0 \\
0 & 0 & \rho_{3} & 0 & 0 & 0 & 0 & 0 & \rho_{2} & 0 & 0 & 0 & 0 & 0 & 0 & 0 \\
0 & 0 & 0 & \rho_{13} & 0 & 0 & \rho_{15} & 0 & 0 & \rho_{10} & 0 & 0 & \rho_{6} & 0 & 0 & 0 \\
0 & 0 & 0 & 0 & 0 & 0 & 0 & 0 & 0 & 0 & \rho_{1} & 0 & 0 & 0 & 0 & 0 \\
0 & 0 & 0 & 0 & 0 & 0 & 0 & 0 & 0 & 0 & 0 & \rho_{8} & 0 & 0 & \rho_{11} & 0 \\
0 & 0 & 0 & \rho_{14} & 0 & 0 & \rho_{12} & 0 & 0 & \rho_{13} & 0 & 0 & \rho_{4} & 0 & 0 & 0 \\
0 & 0 & 0 & 0 & 0 & 0 & 0 & \rho_{11} & 0 & 0 & 0 & 0 & 0 & \rho_{8} & 0 & 0 \\
0 & 0 & 0 & 0 & 0 & 0 & 0 & 0 & 0 & 0 & 0 & \rho_{11} & 0 & 0 & \rho_{8} & 0 \\
0 & 0 & 0 & 0 & 0 & 0 & 0 & 0 & 0 & 0 & 0 & 0 & 0 & 0 & 0 & \rho_{1}
\end{array}\right),
$$

whose elements (Boltzmann weights) are given explicitly in Appendix A.

It satisfies the non-additive Yang-Baxter algebra

$$
R_{12}(u, v) R_{13}(u, w) R_{23}(v, w)=R_{23}(v, w) R_{13}(u, w) R_{12}(u, v)
$$

and enjoys the following properties

$$
\begin{aligned}
& R_{12}(u, v) R_{21}(v, u)=1, \\
& \tilde{R}_{21}^{\mathrm{t}_{1}}(-v, u) R_{12}^{\mathrm{t}_{2}}(u,-v)=1, \\
& \tilde{R}_{12}^{\mathrm{t}_{2}}(-u, v) R_{21}^{\mathrm{t}_{1}}(v,-u)=1 .
\end{aligned}
$$

Above, $\mathrm{t}_{i}$ denotes matrix transposition in the $i$ th space and $R_{21}=P \cdot R_{12} \cdot P$, where $P$ is the permutation operator. The matrix $\tilde{R}$ is a new object introduced in Ref. [27] in order to handle integrable boundary terms. We may take either (2.9) or (2.10) as its definition. We remark here that neither the crossing unitarity nor the difference property is valid for this $R$-matrix, which turns the calculations into a complex problem. 
The Yang-Baxter algebra can also be verified for the monodromy matrix $T$

$$
R_{12}(u, v) \stackrel{1}{T}(u) \stackrel{2}{T}(v)=\stackrel{2}{T}(v) \stackrel{1}{T}(u) R_{12}(u, v),
$$

which is defined, as usual, by

$$
T(u)=L_{0 N}(u) \cdots L_{01}(u) .
$$

Above the subscripts 0 and $N, \ldots, 2,1$ denote the auxiliary and quantum spaces, respectively, and the local Lax operator is given by

$$
L_{j}(u)=\left(\begin{array}{cccc}
P_{j}^{+} Q_{j}^{+} & f_{1}(u) P_{j}^{+} \tau_{j}^{-} & f_{1}(u) \sigma_{j}^{-} \tilde{Q}_{j}^{+} & f_{1}(u) f_{2}(u) \sigma_{j}^{-} \tau_{j}^{-} \\
f_{1}(u) \tilde{P}_{j}^{+} \tau_{j}^{+} & \tilde{P}_{j}^{+} Q_{j}^{-} & f_{2}^{2}(u) \sigma_{j}^{-} \tau_{j}^{+} & f_{2}(u) \sigma_{j}^{-} \tilde{Q}_{j}^{-} \\
f_{1}(u) \sigma_{j}^{+} Q_{j}^{+} & f_{1}^{2}(u) \sigma_{j}^{+} \tau_{j}^{-} & P_{j}^{-} \tilde{Q}_{j}^{+} & f_{2}(u) P_{j}^{-} \tau_{j}^{-} \\
f_{1}(u) f_{2}(u) \sigma_{j}^{+} \tau_{j}^{+} & f_{2}(u) \sigma_{j}^{+} Q_{j}^{-} & f_{2}(u) \tilde{P}_{j}^{-} \tau_{j}^{+} & \tilde{P}_{j} \tilde{Q}^{-}
\end{array}\right),
$$

where

$$
\begin{array}{ll}
P_{j}^{ \pm}=\frac{1}{2}\left(1 \pm \sigma_{j}^{z}\right)+\frac{u}{2} \exp (\beta)\left(1 \mp \sigma_{j}^{z}\right), & \tilde{P}_{j}^{ \pm}=\frac{1}{2}\left(1 \pm \sigma_{j}^{z}\right)+\frac{u}{2}\left(1 \mp \sigma_{j}^{z}\right), \\
Q_{j}^{ \pm}=\frac{1}{2}\left(1 \pm \tau_{j}^{z}\right)+\frac{u}{2} \exp (\beta)\left(1 \mp \tau_{j}^{z}\right), & \tilde{Q}_{j}^{ \pm}=\frac{1}{2}\left(1 \pm \tau_{j}^{z}\right)+\frac{u}{2}\left(1 \mp \tau_{j}^{z}\right), \\
f_{1}(u)=\sqrt{1+u^{2} \exp (2 \beta)}, & f_{2}(u)=\sqrt{1+u^{2}} .
\end{array}
$$

Next, following the QISM adapted to the case of special boundary conditions, we define the doubled monodromy matrix as

$$
U_{-}(u)=T(u) K_{-}(u) T^{-1}(-u),
$$

such that the transfer matrix is given by

$$
\tau(u)=\operatorname{Tr}_{0} K_{+}(u) U_{-}(u) .
$$

Above $T^{-1}$ is the inverse of the monodromy $T$ and $K_{ \pm}$are the matrices defining the boundaries. The requirement that they obey the RE [27]

$$
\begin{aligned}
& R_{12}(u, v) \stackrel{1}{K}-(u) R_{21}(v,-u) \stackrel{2}{K}-(v) \\
& \quad=\stackrel{1}{K}-(v) R_{12}(u,-v) \stackrel{K}{K}-(u) R_{21}(-v,-u), \\
& R_{21}^{\mathrm{t}_{1} \mathrm{t}_{2}}(v, u) \stackrel{1}{\mathrm{t}_{1}}(u) \tilde{R}_{12}(-u, v) \stackrel{2}{\mathrm{t}_{2}}(v) \\
& \quad=\stackrel{2}{\mathrm{t}_{2}}(v) \tilde{R}_{21}(-v, u) \stackrel{1}{\mathrm{t}_{+}^{\mathrm{t}_{1}}}(u) R_{12}^{\mathrm{t}_{1} \mathrm{t}_{2}}(-u,-v)
\end{aligned}
$$

together with the Yang-Baxter algebra assure that the transfer matrix commutes for different spectral parameters, proving the integrability of the model. Therefore, the transfer matrix (2.15) may be considered as the generating function of infinitely many integrals of motion for the system. We emphasize that there is no isomorphism between the matrices $K_{+}(u)$ and $K_{-}(u)$, due to the absence of the crossing symmetry for the $R$ matrix. Therefore, we have to solve the two RE separately in order to fix the boundaries. In Appendix A, this calculation is presented in detail using the variable-separation 
prescription. This approach, which was already proposed in [29] to treat the $D_{2}^{(2)}$ model with $\mathrm{OBC}$, is extended here for the case of an $R$-matrix without the difference property. It is in fact a systematic method which simplifies drastically the calculation of the $K_{ \pm}$-matrices, and also helps in the algebraic nested Bethe ansatz (see next section) We find two different classes of boundary $K_{ \pm}$-matrices consistent with the integrability of the Hamiltonian (2.1), listed below

$$
K_{ \pm}(u)=\left(\begin{array}{cccc}
\mathrm{K} 1_{ \pm}(u) & 0 & 0 & 0 \\
0 & \mathrm{~K} 2_{ \pm}(u) & 0 & 0 \\
0 & 0 & \mathrm{~K} 3_{ \pm}(u) & 0 \\
0 & 0 & 0 & \mathrm{~K} 4_{ \pm}(u)
\end{array}\right) .
$$

Solutions for $K_{-}$, corresponding to the left boundary:

Case I:

$$
\begin{aligned}
& \mathrm{K} 1_{-}(u)=\left(u-c_{1}\right)\left(h^{2} u-c_{1}\right), \\
& \mathrm{K} 2_{-}(u)=\left(c_{1}-u\right)\left(h^{2} u+c_{1}\right), \\
& \mathrm{K} 3_{-}(u)=\left(c_{1}-u\right)\left(h^{2} u+c_{1}\right), \\
& \mathrm{K} 4_{-}(u)=\left(u+c_{1}\right)\left(h^{2} u+c_{1}\right) ;
\end{aligned}
$$

Case II:

$$
\begin{aligned}
& \mathrm{K} 1_{-}(u)=\left(1+c_{1} h u\right), \\
& \mathrm{K} 2_{-}(u)=\left(1-c_{1} h u\right), \\
& \mathrm{K} 3_{-}(u)=\left(1+c_{1} h u\right), \\
& \mathrm{K} 4_{-}(u)=\left(1-c_{1} h u\right),
\end{aligned}
$$

Solutions for $K_{+}$, corresponding to the right boundary:

Case I:

$$
\begin{aligned}
& \mathrm{K} 1_{+}(u)=\left(c_{2} u+1\right)\left(h^{2} c_{2} u+1\right), \\
& \mathrm{K} 2_{+}(u)=h^{2}\left(c_{2} u-1\right)\left(c_{2} u+1\right), \\
& \mathrm{K} 3_{+}(u)=\left(c_{2} u-1\right)\left(c_{2} u+1\right), \\
& \mathrm{K} 4_{+}(u)=\left(c_{2} u-1\right)\left(c_{2} u-h^{2}\right) ;
\end{aligned}
$$

Case II:

$$
\begin{aligned}
& \mathrm{K} 1_{+}(u)=h\left(h u-c_{2}\right), \\
& \mathrm{K} 2_{+}(u)=h\left(h u-c_{2}\right), \\
& \mathrm{K} 3_{+}(u)=\left(u+h c_{2}\right), \\
& \mathrm{K} 4_{+}(u)=\left(u+h c_{2}\right),
\end{aligned}
$$

where $h=\exp (\beta)$. These two classes of boundary $K_{ \pm}$-matrices provide four possible choices of BC, according to the combination of the boundary pairs $\left(K_{+}(u), K_{-}(u)\right)$, which are being labeled by (I, I), (II, II), (I, II) and (II, I) and originate the boundary terms b.t. ${ }^{(I, I)}$, b.t. ${ }^{(\mathrm{II}, \mathrm{II})}$, b.t. ${ }^{\text {(I,II) }}$ and b.t. ${ }^{(\mathrm{II}, \mathrm{I})}$, respectively. Taking into account the fact that $\operatorname{Tr} K_{+}(0)=0$ 
and $\operatorname{Tr} K_{+}^{\prime}(0)=0$ in the cases (I, I) and (I, II) and $\operatorname{Tr} K_{+}(0)=0$ in the cases (II, II) and (II, I), we can show that the Hamiltonian (2.1) with the boundary terms b.t. ${ }^{(\mathrm{I}, \mathrm{I})}$ and b.t. ${ }^{\text {(I,II) }}$ is related to the transfer matrix (2.15) in the following way

$$
\tau(u)=C_{1} u^{2}+C_{2}(H+\text { const }) u^{3}+\cdots,
$$

while

$$
\tau(u)=C_{3} u+C_{4}(H+\text { const }) u^{2}+\cdots
$$

for the Hamiltonian (2.1) with the boundary terms b.t. ${ }^{\text {(II,II) }}$ and b.t. ${ }^{\text {(II,I) }}$. The difference in the leading term expansions (2.23), (2.24) is a consequence of the fact that for case I the matrix $K_{+}(u)$ is quadratic in the variable $u$ while for case II it is linear. Above $C_{i}, i=$ $1, \ldots, 4$, are some scalar functions of the boundary parameters. In the next sections, we shall be focusing in the solution of the eigenvalue problem of the transfer matrix (2.15) and, consequently, in the diagonalization of the present model.

\section{Algebraic Bethe ansatz I: action of the operators on the pseudovacuum}

In order to carry out the algebraic Bethe ansatz for the Bariev model with boundaries, we first need to find the eigenvalues and eigenvectors of the transfer matrix (2.15)

$$
\tau\left|\Phi_{n}\right\rangle=\lambda\left|\Phi_{n}\right\rangle
$$

This will be achieved by extending the Bethe ansatz techniques developed in $[30,31]$ for the Hubbard model to the present case. Following this prescription, the eigenvectors of the transfer matrix are given by

$$
\left|\Phi_{n}\right\rangle=\boldsymbol{\Phi}_{n} \cdot \boldsymbol{F}|0\rangle
$$

where the components of $\boldsymbol{F}$ are coefficients of an arbitrary linear combination of vectors $\boldsymbol{\Phi}_{n}$ (see next section for details) and $|0\rangle$ is the pseudovacuum state, choosen here as the standard ferromagnetic one

$$
|0\rangle=\otimes_{j=1}^{N}|0\rangle_{j},
$$

where

$$
|0\rangle_{i}=\left(\begin{array}{l}
1 \\
0
\end{array}\right)_{i} \otimes\left(\begin{array}{l}
1 \\
0
\end{array}\right)_{i},
$$

which corresponds to the doubly occupied state. In this section we will investigate the action of the monodromy matrices $T, T^{-1}, U_{-}$on this state. We begin by writing the periodic monodromy matrix $T(u)$ as

$$
T(u)=\left(\begin{array}{cccc}
B(u) & B_{1}(u) & B_{2}(u) & F(u) \\
C_{1}(u) & A_{11}(u) & A_{12}(u) & E_{1}(u) \\
C_{2}(u) & A_{21}(u) & A_{22}(u) & E_{2}(u) \\
C_{3}(u) & C_{4}(u) & C_{5}(u) & D(u)
\end{array}\right),
$$


and $\bar{T}(u)=T^{-1}(-u)$ as

$$
\bar{T}(u)=\left(\begin{array}{cccc}
\bar{B}(u) & \bar{B}_{1}(u) & \bar{B}_{2}(u) & \bar{F}(u) \\
\bar{C}_{1}(u) & \bar{A}_{11}(u) & \bar{A}_{12}(u) & \bar{E}_{1}(u) \\
\bar{C}_{2}(u) & \bar{A}_{21}(u) & \bar{A}_{22}(u) & \bar{E}_{2}(u) \\
\bar{C}_{3}(u) & \bar{C}_{4}(u) & \bar{C}_{5}(u) & \bar{D}(u)
\end{array}\right) .
$$

By computing the action of $T(u)$ and $\bar{T}(u)$ on the pseudovacuum we find

$$
\begin{aligned}
& B(u)|0\rangle=\bar{B}(u)|0\rangle=|0\rangle, \quad D(u)|0\rangle=\bar{D}(u)|0\rangle=[u]^{2 N}|0\rangle, \\
& A_{a a}(u)|0\rangle=\bar{A}_{a a}(u)|0\rangle=[u \exp (\beta)]^{N}|0\rangle, \\
& A_{12}(u)|0\rangle=\bar{A}_{12}(u)|0\rangle=0, \quad A_{21}(u)|0\rangle=\bar{A}_{21}(u)|0\rangle=0, \\
& B_{a}(u)|0\rangle \neq 0, \quad \bar{B}_{a}(u)|0\rangle \neq 0, \\
& E_{a}(u)|0\rangle \neq 0, \quad \bar{E}_{a}(u)|0\rangle \neq 0, \\
& F(u)|0\rangle \neq 0, \quad \bar{F}(u)|0\rangle \neq 0, \\
& C_{i}(u)|0\rangle=\bar{C}_{i}(u)|0\rangle=0, \quad i=1, \ldots, 5, \quad a, b=1,2 .
\end{aligned}
$$

Similarly, the doubled monodromy matrix $U_{-}=T(u) K_{-}(u) \bar{T}(u)(2.14)$ can be arranged as

$$
U_{-}(u)=\left(\begin{array}{cccc}
\tilde{B}(u) & \tilde{B}_{1}(u) & \tilde{B}_{2}(u) & \tilde{F}(u) \\
\tilde{C}_{1}(u) & \tilde{A}_{11}(u) & \tilde{A}_{12}(u) & \tilde{E}_{1}(u) \\
\tilde{C}_{2}(u) & \tilde{A}_{21}(u) & \tilde{A}_{22}(u) & \tilde{E}_{2}(u) \\
\tilde{C}_{3}(u) & \tilde{C}_{4}(u) & \tilde{C}_{5}(u) & \tilde{D}(u)
\end{array}\right) .
$$

Then using Eq. (3.31) and the Yang-Baxter algebra

$$
\stackrel{2}{T}(u) R_{12}(u,-u) \stackrel{1}{T}(u)=\stackrel{1}{T}(u) R_{12}(u,-u) \stackrel{2}{T}(u)
$$

we can calculate the action of the doubled monodromy $U_{-}$on the pseudovacuum, which due to the existence of two classes of boundary $K_{-}$-matrices (2.19), (2.20) yields (see Appendix B)

$$
\begin{aligned}
& \tilde{B}(u)|0\rangle=W_{1}^{-}(u) B(u) \bar{B}(u)|0\rangle, \\
& \hat{A}_{a a}(u)|0\rangle=W_{a+1}^{-}(u) A_{a a}(u) \bar{A}_{a a}(u)|0\rangle, \\
& \hat{D}(u)|0\rangle=W_{4}^{-}(u) D(u) \bar{D}(u)|0\rangle, \\
& \tilde{B}_{a}(u)|0\rangle \neq 0, \quad \tilde{E}_{a}(u)|0\rangle \neq 0, \\
& \tilde{A}_{a b}(u)|0\rangle=0, \quad \tilde{F}(u) \neq 0, \\
& \tilde{C}_{i}(u)|0\rangle=0, \quad i=1, \ldots, 5, \quad a \neq b=1,2,
\end{aligned}
$$

where for case I

$$
\begin{aligned}
& W_{1}^{-}(u)=1, \\
& W_{2}^{-}(u)=W_{3}^{-}(u)=-\frac{2 h^{2} u\left(u c_{1}-1\right)}{\left(h^{2} u^{2}-1\right)\left(h^{2} u-c_{1}\right)}, \\
& W_{4}^{-}(u)=\frac{2 u^{2}\left(u c_{1}-1\right)\left(h^{2}+1\right)\left(u c_{1}-h^{2}\right)}{\left(u^{2}-1\right)\left(u^{2}-h^{2}\right)\left(h^{2} u^{2}-c_{1}\right)\left(u-c_{1}\right)}
\end{aligned}
$$


and for case II

$$
\begin{aligned}
& W_{1}^{-}(u)=1, \quad W_{2}^{-}(u)=\frac{2 h^{2} u^{2}}{\left(h^{2} u^{2}-1\right)}, \\
& W_{3}^{-}(u)=\frac{2 h u\left(h u+c_{1}\right)}{\left(h^{2} u^{2}-1\right)\left(1+c_{1} h u\right)}, \quad W_{4}^{-}(u)=\frac{2 u^{3}\left(h^{2}+1\right)\left(u+h c_{1}\right)}{\left(u^{2}-1\right)\left(u^{2}-h^{2}\right)\left(1+c_{1} h u\right)} .
\end{aligned}
$$

Actually, in order to considerably simplify the calculations, the following transformations

$$
\begin{aligned}
& \hat{A}_{a a}(u)=\tilde{A}_{a a}(u)-\frac{\rho_{2}(u,-u)}{\rho_{1}(u,-u)} \tilde{B}(u), \\
& \hat{D}(u)=\tilde{D}(u)-\frac{\rho_{4}(u,-u)}{\rho_{1}(u,-u)} \tilde{B}(u)-\frac{1+u^{2}}{h^{2}-u^{2}} \hat{A}_{11}(u)-\frac{h^{2}\left(1+u^{2}\right)}{h^{2}-u^{2}} \hat{A}_{22}(u),
\end{aligned}
$$

were used in the above Eqs. (3.34)-(3.36). In fact, these types of transformations have also been performed in other models with $\mathrm{OBC}[5,9,10,12,13]$. They simplify not only these expressions for the action of the operators on the pseudovacuum, but also simplify the fundamental commutation relations, allowing for an easy recognition of the "wanted" and "unwanted" terms, essential to the Bethe ansatz machinery (see next section for details). Then, after this transformation and taking into account the two sets of boundary matrices $K_{+}$(2.21), (2.22), the transfer matrix (2.15) can be recast as

$$
\tau(u)=W_{1}^{+}(u) \tilde{B}(u)+\sum_{a=1}^{2} W_{a+1}^{+}(u) \hat{A}_{a a}(u)+W_{4}^{+}(u) \hat{D}(u),
$$

where for case I

$$
\begin{aligned}
W_{1}^{+}(u) & =\frac{2 u^{2}\left(u-c_{2}\right)\left(h^{2}+1\right)\left(h^{2} u-c_{2}\right)}{\left(u c_{2}+1\right)\left(u h^{2} c_{2}+1\right)\left(u^{2}-1\right)\left(h^{2} u^{2}-1\right)}, \\
W_{2}^{+}(u) & =\frac{u\left(u c_{2}-1\right)\left(u-c_{2}\right)\left(h^{2}+1\right)}{\left(h^{2} u c_{2}+1\right)\left(u^{2}-h^{2}\right)\left(u c_{2}+1\right)}, \\
W_{3}^{+}(u) & =\frac{h^{2} u\left(u c_{2}-1\right)\left(u-c_{2}\right)\left(h^{2}+1\right)}{\left(h^{2} u c_{2}+1\right)\left(u^{2}-h^{2}\right)\left(u c_{2}+1\right)}, \\
W_{4}^{+}(u) & =\frac{\left(u c_{2}-1\right)\left(u c_{2}-h^{2}\right)}{\left(u c_{2}+1\right)\left(h^{2} u c_{2}+1\right)},
\end{aligned}
$$

and for case II

$$
\begin{aligned}
W_{1}^{+}(u) & =\frac{2 u\left(h^{2}+1\right)\left(h c_{2} u+1\right)}{h\left(u^{2}-1\right)\left(h^{2} u^{2}-1\right)\left(h u-c_{2}\right)}, \\
W_{2}^{+}(u) & =-\frac{\left(h^{2}+1\right)\left(u+h c_{2}\right)}{h\left(u^{2}-h^{2}\right)\left(h u-c_{2}\right)}, \\
W_{3}^{+}(u) & =-\frac{u\left(h^{2}+1\right)\left(h+u c_{2}\right)}{\left(u^{2}-h^{2}\right)\left(h u-c_{2}\right)}, \quad W_{4}^{+}(u)=\frac{\left(u+h c_{2}\right)}{h\left(h u-c_{2}\right)} .
\end{aligned}
$$

Notice from Eqs. (3.34)-(3.37) that the Lax operators (3.32) act as creation (annihilation) operators on the pseudovacuum. Before ending this section, we would like to recall that the boundary matrix pairs $\left(K_{+}(u), K_{-}(u)\right)$ lead to four kinds of boundary fields preserving 
the integrability of the model. Consequently, the factors $W_{i}^{ \pm}(k) i=1, \ldots, 4$ also form a pair $\left(W_{i}^{+}(k), W_{i}^{-}(k)\right)$ leading to four possible choices consistent with the boundary matrix pairs. The perfect factorization provide us with the nesting boundary $K$-matrices appearing in (3.35) and (3.39), respectively. In the following section, we shall prove that they indeed constitute the solutions of the RE for the hidden asymmetric $X X Z$ spin open chain.

\section{Algebraic Bethe ansatz II: the nesting procedure}

The next step in the solution of the eigenvalue problem of the transfer matrix consists in the computation of the necessary commutation relations between the diagonal fields and the creation fields. From the RE (2.16) and the definition (3.32), after tedious calculations, we obtain the main fundamental commutation relations. We display below just those needed for future calculations

$$
\begin{aligned}
& \tilde{B}(u) \tilde{B}_{a}(v)=\frac{(u+v)\left(u v h^{2}+1\right)}{(u-v)\left(u v h^{2}-1\right)} \tilde{B}_{a}(v) \tilde{B}(u)+\text { u.t. }, \\
& \begin{aligned}
\hat{D}(u) \tilde{B}_{a}(v)=\frac{(u+v)\left(u v h^{2}+1\right)}{\left(u-v h^{2}\right)(u v-1)} \tilde{B}_{a}(v) \hat{D}(u)+\text { u.t., } \\
\hat{A}_{a d_{1}}(u) \tilde{B}_{d_{2}}(v)=\frac{(u+v)\left(1+u v h^{2}\right)}{(u-v)\left(u v h^{2}-1\right)} \bar{r}_{12}(u,-v)_{a_{1} c_{2}}^{c_{1} b_{2}} \bar{r}_{21}(-v,-u)_{b_{1} b_{2}}^{d_{1} d_{2}} \\
\quad \times \tilde{B}_{c_{2}}(v) \hat{A}_{c_{1} b_{1}}(u)+\text { u.t. },
\end{aligned} \\
& \tilde{B}_{a}(u) \otimes \tilde{B}_{b}(v) \quad \begin{array}{l}
1-u v \\
=\left\{\tilde{B}_{c}(v) \otimes \tilde{B}_{d}(u)+\frac{\sqrt{1+h^{2} u^{2}} \sqrt{1+v^{2}}}{1-u}(v) \vec{\xi}(I \otimes \tilde{A}(u))\right\} . r(-v,-u)
\end{array} \\
& \quad-\frac{\sqrt{1+h^{2} v^{2}} \sqrt{1+u^{2}} \tilde{F}(u) \vec{\xi}(I \otimes \tilde{A}(v))}{1-u v} \\
& \quad+\frac{\left(h^{2} u+v\right) \sqrt{1+h^{2} u^{2}} \sqrt{1+v^{2}}}{\left(u-h^{2} v\right)(u v-1)} \\
& \quad \times\left[\tilde{F}(v) \tilde{B}(u)-\frac{\left(h^{2} v+u\right) \sqrt{1+h^{2} v^{2}} \sqrt{1+u^{2}}}{\left(h^{2} u+v\right) \sqrt{1+h^{2} u^{2}} \sqrt{1+v^{2}}} \tilde{F}(u) \tilde{B}(v)\right] . \vec{\xi},
\end{aligned}
$$

where

$$
\begin{gathered}
\vec{\xi}=\left(0, h^{-1}, 1,0\right), \quad \tilde{A}(u)=\left(\begin{array}{ccc}
\tilde{A}_{11}(u) & \tilde{A}_{12}(u) \\
\tilde{A}_{21}(u) & \tilde{A}_{22}(u)
\end{array}\right), \\
\bar{r}_{12}(u,-v)=\left(\begin{array}{cccc}
1 & 0 & 0 & 0 \\
0 & \bar{c}(u,-v) & \bar{d}(u,-v) & 0 \\
0 & \bar{a}(u,-v) & \bar{c}(u,-v) & 0 \\
0 & 0 & 0 & 1
\end{array}\right),
\end{gathered}
$$




$$
r(-v,-u)=\left(\begin{array}{cccc}
1 & 0 & 0 & 0 \\
0 & a(-v,-u) & c(-v,-u) & 0 \\
0 & c(-v,-u) & d(-v,-u) & 0 \\
0 & 0 & 0 & 1
\end{array}\right)
$$

and

$$
\begin{aligned}
& \bar{a}(u,-v)=\frac{\rho_{7}(u,-v) \rho_{1}(u,-v)-\rho_{2}^{2}(u,-v)}{\rho_{1}^{2}(u,-v)-\rho_{2}^{2}(u,-v)}, \\
& \bar{d}(u,-v)=\frac{\rho_{10}(u,-v) \rho_{1}(u,-v)-\rho_{2}^{2}(u,-v)}{\rho_{1}^{2}(u,-v)-\rho_{2}^{2}(u,-v)}, \\
& \bar{c}(u,-v)=\frac{\rho_{1}(u,-v) \rho_{15}(u,-v)}{\rho_{1}^{2}(u,-v)-\rho_{2}^{2}(u,-v)} \\
& a(-v,-u)=\frac{\rho_{14}(-v,-u) \rho_{10}(-v,-u)-\rho_{13}^{2}(-v,-u)}{\rho_{1}(-v,-u) \rho_{14}(-v,-u)}, \\
& d(-v,-u)=\frac{\rho_{14}(-v,-u) \rho_{7}(-v,-u)-\rho_{12}^{2}(-v,-u)}{\rho_{1}(-v,-u) \rho_{14}(-v,-u)}, \\
& c(-v,-u)=\frac{\rho_{14}(-v,-u) \rho_{15}(-v,-u)-\rho_{12}(-v,-u) \rho_{13}(-v,-u)}{\rho_{1}(-v,-u) \rho_{14}(-v,-u)} .
\end{aligned}
$$

It remains to consider the commutation relations for the creation field $\tilde{F}$. Since they are extremely involved, we have collected them in Appendix B. We remark here that the fundamental commutation relations are much more complicated in comparison with those obtained in the case of periodic boundary conditions, turning the calculations here much more cumbersome and difficult to treat. In all relations, we have omitted the unwanted terms (those that can not generate an eigenvector of the transfer matrix) to save space. It turns out that the auxiliary matrices $\bar{r}_{12}(u,-v)$ and $r(-v,-u)$ are nothing but the rational $R$-matrices of an isotropic asymmetric six-vertex model. Indeed, by performing the parameterization $u=\exp \left(\mathrm{i} k_{1}\right), v=\exp \left(\mathrm{i} k_{2}\right)$ and $h=\exp (\beta)=\exp (-\mathrm{i} \eta)$, one may present these matrices as

$$
\begin{aligned}
& r(-v,-u)=r\left(k_{1}-k_{2}\right)=\left(\begin{array}{cccc}
1 & 0 & 0 & 0 \\
0 & a\left(k_{1}-k_{2}\right) & c\left(k_{1}-k_{2}\right) & 0 \\
0 & c\left(k_{1}-k_{2}\right) & d\left(k_{1}-k_{2}\right) & 0 \\
0 & 0 & 0 & 1
\end{array}\right), \\
& \bar{r}_{12}(u,-v)=P \cdot r\left(k_{1}+k_{2}-2 \eta\right), \\
& \bar{r}_{21}(u,-v)=P \cdot r\left(k_{1}-k_{2}\right) . P
\end{aligned}
$$

with the Boltzmann weights

$$
\begin{aligned}
& a(k)=\frac{\sin \eta}{\sin \left(\frac{k}{2}+\eta\right)} \exp \left(-\mathrm{i} \frac{1}{2} k\right), \\
& d(k)=\frac{\sin \eta}{\sin \left(\frac{k}{2}+\eta\right)} \exp \left(\mathrm{i} \frac{1}{2} k\right),
\end{aligned}
$$




$$
c(k)=-\frac{\sin \frac{k}{2}}{\sin \left(\frac{k}{2}+\eta\right)} \exp \left(-\mathrm{i} \frac{k}{2}\right) .
$$

In view of the commutation relation (4.43), we may phenomenologically write [30] the $n$-particle state as

$$
\left|\Phi_{n}\left(k_{1}, \ldots, k_{n}\right)\right\rangle=\Phi_{n}\left(k_{1}, \ldots, k_{n}\right) \boldsymbol{F}|0\rangle,
$$

with a recursive relation of $n$-particle vector

$$
\begin{aligned}
& \Phi_{n}\left(k_{1}, \ldots, k_{n}\right)= \tilde{B}_{a_{1}}\left(k_{1}\right) \otimes \Phi_{n-1}\left(k_{2}, \ldots, k_{n}\right) \\
&++\sum_{j=2}^{n}\left\{\left[\vec{\xi} \otimes \tilde{F}\left(k_{1}\right)\right] \Phi_{n-2}\left(k_{2}, \ldots, k_{j-1}, k_{j+1}, \ldots, k_{n}\right)\right. \\
&\left.\times \tilde{B}\left(k_{j}\right) G_{j-1}^{(n)}\left(k_{1}, \ldots, k_{n}\right)\right\} \\
&+\sum_{j=2}^{n}\left\{\left[\vec{\xi} \otimes \tilde{F}\left(k_{1}\right)\right] \Phi_{n-2}\left(k_{2}, \ldots, k_{j-1}, k_{j+1}, \ldots, k_{n}\right)\right. \\
&\left.\times\left[I \otimes \tilde{A}\left(k_{j}\right)\right] H_{j-1}^{(n)}\left(k_{1}, \ldots, k_{n}\right)\right\} .
\end{aligned}
$$

Above $\boldsymbol{F}=F^{a_{1}, \ldots, a_{n}}$ are the coefficients of arbitrary linear combination of the vectors reflecting the 'spin' degrees of freedom with $a_{i}=1,2(i=1, \ldots, n)$ and $\vec{\xi}$ plays the role of forbidding two spin up or two spin down particles at the same site. $\tilde{F}(u)$ creates a local particle pair with opposite spins. From the commutation relation (4.43), we can also conclude that $\Phi_{n}\left(k_{1}, \ldots, k_{n}\right)$ satisfies the symmetry relation

$$
\begin{aligned}
& \Phi_{n}\left(k_{1}, \ldots, k_{j}, k_{j+1}, \ldots, k_{n}\right) \\
& \quad=\Phi_{n}\left(k_{1}, \ldots, k_{j+1}, k_{j}, \ldots, k_{n}\right) . r\left(k_{j}-k_{j+1}\right) .
\end{aligned}
$$

This symmetry giving a restriction to the functions $H_{j-1}^{(n)}\left(k_{1}, \ldots, k_{n}\right)$ and $G_{j-1}^{(n)}\left(k_{1}, \ldots, k_{n}\right)$ is very useful to deduce the coefficients and simplify the unwanted terms in the eigenvalue of the transfer matrix. In fact, after performing three-particle scattering, the explicit form of these coefficients can be fixed. However checking three-particle scattering is indeed a extremely tough calculation and we had to leave the coefficients to be determined later. Explicitly, we display the two-particle state

$$
\begin{aligned}
\Phi_{2}\left(k_{1}, k_{2}\right)= & \tilde{B}_{a_{1}}\left(k_{1}\right) \otimes \tilde{B}_{a_{2}}\left(k_{2}\right) \\
& +\frac{\sqrt{1+e^{\mathrm{i} 2 k_{1}}} \sqrt{1+e^{\mathrm{i} 2\left(k_{2}-\eta\right)}}}{1-e^{\mathrm{i}\left(k_{1}+k_{2}\right)}} \tilde{F}\left(k_{1}\right) \vec{\xi}\left(I \otimes \tilde{A}\left(k_{2}\right)\right) \\
& +\frac{\cos \left(\frac{k_{1}-k_{2}}{2}+\eta\right) \sqrt{1+e^{\mathrm{i} 2 k_{1}}} \sqrt{1+e^{\mathrm{i} 2\left(k_{2}-\eta\right)}}}{\sin \left(\frac{k_{1}-k_{2}}{2}+\eta\right)\left(1-e^{\mathrm{i} 2\left(k_{1}+k_{2}\right)}\right)} \tilde{F}\left(k_{1}\right) \tilde{B}\left(k_{2}\right) . \vec{\xi} .
\end{aligned}
$$

Implementing a rescaling $k \rightarrow k+\eta$ and setting $c_{1}=e^{\mathrm{i} 2 \xi_{-}}, c_{2}=e^{\mathrm{i} 2 \xi_{+}}$, the eigenvalue of the transfer matrix (2.15) acting on the state (4.54) can be given as 


$$
\begin{aligned}
& \tau(k)\left|\Phi_{n}\left(k_{1}, \ldots, k_{n}\right)\right\rangle \\
& =\left\{W_{1}^{+}(k) W_{1}^{-}(k) \prod_{i=1}^{n} \frac{\cos \left(\frac{k+k_{i}}{2}\right) \cos \left(\frac{k-k_{i}}{2}\right)}{\sin \left(\frac{k+k_{i}}{2}\right) \sin \left(\frac{k-k_{i}}{2}\right)}\right. \\
& \quad+\sum_{a=1}^{2} W_{a+1}^{+}(k) W_{a+1}^{-}(k) e^{\mathrm{i} 2 N k} \prod_{i=1}^{n} \frac{\cos \left(\frac{k+k_{i}}{2}\right) \cos \left(\frac{k-k_{i}}{2}\right)}{\sin \left(\frac{k+k_{i}}{2}\right) \sin \left(\frac{k-k_{i}}{2}\right)} \Lambda_{a a}^{(1)}\left(k,\left\{k_{i}\right\},\left\{\lambda_{j}\right\}\right) \\
& \left.\quad+W_{4}^{+}(k) W_{4}^{-}(k) e^{\mathrm{i} 4 N(k+\eta)} \prod_{i=1}^{n} \frac{\cos \left(\frac{k+k_{i}}{2}\right) \cos \left(\frac{k-k_{i}}{2}\right)}{\sin \left(\frac{k+k_{i}}{2}+\eta\right) \sin \left(\frac{k-k_{i}}{2}+\eta\right)}\right\}\left|\Phi_{n}\left(k_{1}, \ldots, k_{n}\right)\right\rangle
\end{aligned}
$$

provided that

$$
\left.\frac{W_{1}^{+}(k) W_{1}^{-}(k)}{W_{2}^{+}(k) W_{2}^{-}(k) \exp (\mathrm{i} 2 N k)}\right|_{k=k_{i}}=-\Lambda^{(1)} \quad\left(k=k_{i},\left\{k_{i}\right\},\left\{\lambda_{j}\right\}\right), \quad i=1, \ldots, n .
$$

Here $\Lambda^{(1)}\left(\tilde{k},\left\{k_{i}\right\},\left\{\lambda_{j}\right\}\right)$ is the eigenvalue of the reduced transfer matrix $\tau^{(1)}(k)$, which turns out to be an inhomogenous isotropic six-vertex model with open boundary conditions, namely,

$$
\tau^{(1)}(k) F^{a_{1} \cdots a_{n}}=\Lambda^{(1)}\left(k,\left\{k_{i}\right\},\left\{\lambda_{j}\right\}\right) F^{a_{1} \cdots a_{n}},
$$

where

$$
\tau^{(1)}(k)=\operatorname{Tr}_{0} K_{+}^{(1)}(k) T^{(1)}(k) K_{-}^{(1)}(k) T^{(1)^{-1}}(-k) .
$$

The nesting monodromy matrices $T^{(1)}(k)$ and $T^{(1)^{-1}}(-k)$ read

$$
\begin{aligned}
& T^{(1)}(k)=r_{12}\left(k+k_{1}\right)_{a c_{1}}^{a_{1} e_{1}} r_{12}\left(k+k_{2}\right)_{a_{1} c_{2}}^{a_{2} e_{2}} \cdots r_{12}\left(k+k_{n}\right)_{a_{n-1} c_{n}}^{a_{n} e_{n}}, \\
& T^{(1)^{-1}}(-k)=r_{21}\left(k-k_{n}\right)_{b_{n} e_{n}}^{b_{n-1} d_{n}} \cdots r_{21}\left(k-k_{2}\right)_{b_{2} e_{2}}^{b_{1} d_{2}} r_{21}\left(k-k_{1}\right)_{b_{1} e_{1}}^{a d_{1}},
\end{aligned}
$$

and the nesting $K_{ \pm}^{(1)}(k)$ may be chosen as

Case I:

$$
K_{+}^{(1)}(k)=\left(\begin{array}{cc}
e^{\mathrm{i} \eta} & 0 \\
0 & e^{-\mathrm{i} \eta}
\end{array}\right)
$$

in such a way that the factors $W^{+}$can be rewritten as

$$
\begin{aligned}
& W_{1}^{+}(k)=\frac{\sin \left(\frac{k}{2}+\frac{\eta}{2}-\xi_{+}\right) \sin \left(\frac{k}{2}-\frac{\eta}{2}-\xi_{+}\right) \cos \eta}{\cos \left(\frac{k}{2}+\frac{\eta}{2}+\xi_{+}\right) \cos \left(\frac{k}{2}-\frac{\eta}{2}+\xi_{+}\right) \sin k \sin (k+\eta)}, \\
& W_{2}^{+}(k)=W_{3}^{+}(k)=\frac{\sin \left(\frac{k}{2}+\frac{\eta}{2}+\xi_{+}\right) \sin \left(\frac{k}{2}+\frac{\eta}{2}-\xi_{+}\right) \cos \eta}{\cos \left(\frac{k}{2}-\frac{\eta}{2}+\xi_{+}\right) \cos \left(\frac{k}{2}+\frac{\eta}{2}+\xi_{+}\right) \sin (k+2 \eta)}, \\
& W_{4}^{+}(k)=\frac{\sin \left(\frac{k}{2}+\frac{\eta}{2}+\xi_{+}\right) \sin \left(\frac{k}{2}+\frac{3 \eta}{2}+\xi_{+}\right)}{\cos \left(\frac{k}{2}+\frac{\eta}{2}+\xi_{+}\right) \cos \left(\frac{k}{2}-\frac{\eta}{2}+\xi_{+}\right)}
\end{aligned}
$$

and case I:

$$
K_{-}^{(1)}(k)=\left(\begin{array}{cc}
1 & 0 \\
0 & 1
\end{array}\right)
$$


with

$$
\begin{aligned}
& W_{1}^{-}(k)=1, \\
& W_{2}^{-}(k)=W_{3}^{-}(k)=-\frac{\sin \left(\frac{k}{2}+\frac{\eta}{2}+\xi_{-}\right)}{\sin \left(\frac{k}{2}-\frac{\eta}{2}-\xi_{-}\right) \sin k}, \\
& W_{4}^{-}(k)=\frac{\sin \left(\frac{k}{2}+\frac{\eta}{2}+\xi_{-}\right) \sin \left(\frac{k}{2}+\frac{3 \eta}{2}+\xi_{-}\right) \cos \eta}{\sin \left(\frac{k}{2}-\frac{\eta}{2}-\xi_{-}\right) \sin \left(\frac{k}{2}+\frac{\eta}{2}-\xi_{-}\right) \sin (k+\eta) \sin (k+2 \eta)} ;
\end{aligned}
$$

Case II:

$$
K_{+}^{(1)}(k)=\left(\begin{array}{cc}
\cos \left(\frac{k}{2}+\eta-\xi_{+}\right) e^{-\mathrm{i} k} & 0 \\
0 & \cos \left(\frac{k}{2}+\eta+\xi_{+}\right)
\end{array}\right)
$$

with

$$
\begin{aligned}
W_{1}^{+}(k) & =\frac{\cos \left(\frac{k}{2}+\xi_{+}\right) \cos \eta e^{-i k}}{\sin \left(\frac{k}{2}-\xi_{+}\right) \sin k \sin (k+\eta)}, \\
W_{2}^{+}(k) & =W_{3}^{+}(k)=-\frac{\cos \eta}{\sin \left(\frac{k}{2}-\xi_{+}\right) \sin (k+2 \eta)}, \\
W_{4}^{+}(k) & =\frac{\cos \left(\frac{k}{2}+\eta-\xi_{+}\right) e^{i \eta}}{\sin \left(\frac{k}{2}-\xi_{+}\right)},
\end{aligned}
$$

and case II:

$$
K_{-}^{(1)}(k)=\left(\begin{array}{cc}
\cos \left(\frac{k}{2}+\xi_{-}\right) e^{i k} & 0 \\
0 & \cos \left(\frac{k}{2}-\xi_{-}\right)
\end{array}\right)
$$

with

$$
\begin{aligned}
& W_{1}^{-}(k)=1 \\
& W_{2}^{-}(k)=W_{3}^{-}(k)=\frac{1}{\sin k \cos \left(\frac{k}{2}+\xi_{-}\right)}, \\
& W_{4}^{-}(k)=\frac{\cos \left(\frac{k}{2}+\eta-\xi_{-}\right) \cos \eta e^{\mathrm{i}(k+\eta)}}{\cos \left(\frac{k}{2}+\xi_{-}\right) \sin (k+\eta) \sin (k+2 \eta)} .
\end{aligned}
$$

We notice that the nesting boundary $K_{ \pm}^{(1)}(k)$-matrices also constitute four classes of BC consistent with the boundary matrix pairs $\left(K^{+}(k), K^{-}(k)\right)$. So far, the eigenvalue problem of the 1D Bariev model with four kinds of possible boundary fields reduces to the solution of the nested auxiliary transfer matrix (4.60), which can be associated with an inhomogeneous isotropic six-vertex model with open boundary conditions (4.64)-(4.76). Furthermore, we can check that the $r$-matrix (4.48) and the nesting $K_{ \pm}^{(1)}$-matrices (4.64)-(4.76), which arises from the factorization of the charge degree of freedom, indeed satisfy the Yang-Baxter algebra

$$
r_{12}(k-\lambda) \stackrel{1}{T}^{(1)}(k) \stackrel{2}{T}(1)(\lambda)=\stackrel{2}{T}(1)(\lambda) \stackrel{1}{T}^{(1)}(k) r_{12}(k-\lambda)
$$

and the reflection equations 


$$
\begin{aligned}
& r_{12}(k-\lambda) \stackrel{1}{K_{-}^{(1)}}(k) r_{21}(k+\lambda) \stackrel{2}{K_{-}^{(1)}}(\lambda) \\
& =\stackrel{2}{K}(1)(\lambda) r_{12}(k+\lambda) \stackrel{1}{K}(1)(k) r_{21}(k-\lambda), \\
& r_{21}^{\mathrm{t}_{1} \mathrm{t}_{2}}(\lambda-k) \stackrel{1}{K_{+}^{(1)}}{ }^{\mathrm{t}_{1}}(k) \tilde{r}_{12}(-k-\lambda) \stackrel{2}{K}(1) \mathrm{t}_{+}(\lambda) \\
& =\stackrel{2}{K_{+}^{(1)}} \mathrm{t}_{2}(\lambda) \tilde{r}_{21}(-k-\lambda) \stackrel{1}{K}{ }_{+}^{(1)} \mathrm{t}_{1}(k) r_{12}^{\mathrm{t}_{1} \mathrm{t}_{2}}(\lambda-k),
\end{aligned}
$$

where

$$
\begin{aligned}
& r_{12}(k) r_{21}(-k)=1, \\
& \tilde{r}_{21}^{\mathrm{t}_{1}}(-k) r_{12}^{\mathrm{t}_{2}}(k)=1, \\
& \tilde{r}_{12}^{\mathrm{t}_{2}}(-k) r_{21}^{\mathrm{t}_{1}}(k)=1 .
\end{aligned}
$$

Following all steps in solving $X X Z$ open chain in [14], one can completely diagonalize the nesting transfer matrix (4.61) with the help of the main ingredients (4.80)-(4.82) describing the open $\mathrm{BC}$ for the hidden asymmetric $X X Z$ open chain. Therefore, what follows is the exact solution of the $X X Z$ open chain characterizing the spin degree of freedom, i.e.,

$$
\begin{aligned}
\Lambda^{(1)}\left(k,\left\{k_{i}\right\},\left\{\lambda_{j}\right\}\right)\left|\Phi^{(1)}\left(\lambda_{1}, \ldots, \lambda_{M}\right)\right\rangle \\
=\left\{\frac{\sin (k+2 \eta)}{\sin (k+\eta)} W_{11}^{+}(k) W_{11}^{-}(k) A^{(1)}(k) \prod_{j=1}^{M} \frac{\sin \left(\frac{k+\lambda_{j}}{2}\right) \sin \left(\frac{k-\lambda_{j}}{2}-\eta\right)}{\sin \left(\frac{k+\lambda_{j}}{2}+\eta\right) \sin \left(\frac{k-\lambda_{j}}{2}\right)}\right. \\
\left.\quad+\frac{\sin k}{\sin (k+\eta)} W_{22}^{+}(k) W_{22}^{-}(k) D^{(1)}(k) \prod_{j=1}^{M} \frac{\sin \left(\frac{k+\lambda_{j}}{2}+2 \eta\right) \sin \left(\frac{k-\lambda_{j}}{2}+\eta\right)}{\sin \left(\frac{k+\lambda_{j}}{2}+\eta\right) \sin \left(\frac{k-\lambda_{j}}{2}\right)}\right\} \\
\quad \times\left|\Phi^{(1)}\left(\lambda_{l}, \ldots, \lambda_{M}\right)\right\rangle,
\end{aligned}
$$

provided that

$$
\frac{W_{11}^{+}(k) W_{11}^{-}(k) A^{(1)}(k)}{W_{22}^{+}(k) W_{22}^{-}(k) D^{(1)}(k)}=\prod_{\substack{l=1, l \neq j}}^{M} \frac{\sin \left(\frac{\lambda_{j}+\lambda_{l}}{2}+2 \eta\right) \sin \left(\frac{\lambda_{j}-\lambda_{l}}{2}+\eta\right)}{\sin \left(\frac{\lambda_{j}+\lambda_{l}}{2}\right) \sin \left(\frac{\lambda_{j}-\lambda_{l}}{2}-\eta\right)},
$$

where

$$
\begin{aligned}
& A^{(1)}(k)\left|\Phi^{(1)}\right\rangle=\left|\Phi^{(1)}\right\rangle, \\
& D^{(1)}(k)\left|\Phi^{(1)}\right\rangle=\left\{\prod_{i=1}^{n} \frac{\sin \left(\frac{k+\lambda_{i}}{2}\right) \sin \left(\frac{k-\lambda_{i}}{2}\right)}{\sin \left(\frac{k+\lambda_{i}}{2}+\eta\right) \sin \left(\frac{k-\lambda_{i}}{2}+\eta\right)}\right\}\left|\Phi^{(1)}\right\rangle,
\end{aligned}
$$

with case I:

$$
\begin{aligned}
& W_{11}^{+}(k)=W_{11}^{-}(k)=1, \\
& W_{22}^{+}(k)=W_{22}^{-}(k)=1,
\end{aligned}
$$

and case II: 


$$
\begin{aligned}
& W_{11}^{+}(k)=\cos \left(\frac{k}{2}-\xi_{+}\right) e^{-\mathrm{i} k}, \quad W_{11}^{-}(k)=\cos \left(\frac{k}{2}+\xi_{-}\right) e^{\mathrm{i} k}, \\
& W_{22}^{+}(k)=\cos \left(\frac{k}{2}+\eta+\xi_{+}\right) e^{\mathrm{i} \eta}, \quad W_{22}^{-}(k)=\cos \left(\frac{k}{2}+\eta-\xi_{-}\right) e^{-i \eta},
\end{aligned}
$$

respectively.

Finally, making a shift on the spin rapidity $\lambda_{i} \rightarrow \lambda_{i}-\eta$, the eigenvalue of the transfer matrix (2.15) with two classes of boundary $K$-matrices can be given explicitly (up to a common factor)

$$
\begin{aligned}
\tau(u) \mid & \left.\Phi_{n}\left(k_{1}, \ldots, k_{n}\right)\right\rangle \\
= & \left\{\prod _ { i = 1 } ^ { n } \frac { \operatorname { c o s } ( \frac { k + k _ { i } } { 2 } ) \operatorname { c o s } ( \frac { k - k _ { i } } { 2 } ) } { \operatorname { s i n } ( \frac { k + k _ { i } } { 2 } ) \operatorname { s i n } ( \frac { k - k _ { i } } { 2 } ) } \left\{W_{1}^{+}(k) W_{1}^{-}(k)\right.\right. \\
& \left.+\frac{\sin (k+2 \eta)}{\sin (k+\eta)} W_{2}^{+}(k) W_{2}^{-}(k) W_{11}^{+}(k) W_{11}^{-}(k) e^{\mathrm{i} 2 N k} \prod_{l=1}^{M} \frac{\sin \left(\frac{k+\lambda_{l}-\eta}{2}\right) \sin \left(\frac{k-\lambda_{l}-\eta}{2}\right)}{\sin \left(\frac{k+\lambda_{l}+\eta}{2}\right) \sin \left(\frac{k-\lambda_{l}+\eta}{2}\right)}\right\} \\
& +\prod_{i=1}^{n} \frac{\cos \left(\frac{k+k_{i}}{2}\right) \cos \left(\frac{k-k_{i}}{2}\right)}{\sin \left(\frac{k+k_{i}}{2}+\eta\right) \sin \left(\frac{k-k_{i}}{2}+\eta\right)}\left\{W_{4}^{+}(k) W_{4}^{-}(k) e^{\mathrm{i} 4 N(k+\eta)}\right. \\
& +\frac{\sin k}{\sin (k+\eta)} W_{3}^{+}(k) W_{3}^{-}(k) W_{22}^{+}(k) W_{22}^{-}(k) e^{\mathrm{i} 2 N k} \\
& \left.\times \prod_{l=1}^{M} \frac{\sin \left(\frac{k+\lambda_{l}+3 \eta}{2}\right) \sin \left(\frac{k-\lambda_{l}+3 \eta}{2}\right)}{\sin \left(\frac{k+\lambda_{l}+\eta}{2}\right) \sin \left(\frac{k-\lambda_{l}+\eta}{2}\right)}\right\}\left|\Phi_{n}\left(k_{1}, \ldots, k_{n}\right)\right\rangle,
\end{aligned}
$$

where all factorized coefficients $W_{i}^{ \pm}(i=1, \ldots, 4,11$ and 22) have been given explicitly in the above equations, which provide four kinds of possible exact solutions. Finally, the expression above is in fact the eigenvalue of the transfer matrix, if the following Bethe ansatz equations are satisfied for the charge and spin rapidities $k_{i}, \lambda_{j}$ :

$$
\begin{gathered}
e^{\mathrm{i} 2 N k_{i}} \zeta_{1}\left(k_{i}, \xi_{+}\right) \zeta_{2}\left(k_{i}, \xi_{-}\right)=\prod_{l=1}^{M} \frac{\sin \left(\frac{k_{i}+\lambda_{l}+\eta}{2}\right) \sin \left(\frac{k_{i}-\lambda_{l}+\eta}{2}\right)}{\sin \left(\frac{k_{i}+\lambda_{l}-\eta}{2}\right) \sin \left(\frac{k_{i}-\lambda_{l}-\eta}{2}\right)}, \\
\psi_{1}\left(\lambda_{j}, \xi_{+}\right) \psi_{2}\left(\lambda_{j}, \xi_{-}\right) \prod_{l=1}^{n} \frac{\sin \left(\frac{\lambda_{j}+k_{l}+\eta}{2}\right) \sin \left(\frac{\lambda_{j}-k_{l}+\eta}{2}\right)}{\sin \left(\frac{\lambda_{j}+k_{l}-\eta}{2}\right) \sin \left(\frac{\lambda_{j}-k_{l}-\eta}{2}\right)} \\
\left.=\prod_{\substack{l=1 \\
l \neq j}}^{M} \frac{\sin \left(\frac{\lambda_{j}+\lambda_{l}}{2}+\eta\right) \sin \left(\frac{\lambda_{j}-\lambda_{l}}{2}+\eta\right)}{2}-\eta\right) \sin \left(\frac{\lambda_{j}-\lambda_{l}}{2}-\eta\right) \\
j=1, \ldots, M, \quad i=1, \ldots, n .
\end{gathered}
$$

The expressions for $\zeta_{a}\left(k_{i}, \xi_{ \pm}\right)$and $\psi_{a}\left(\lambda_{j}, \xi_{ \pm}\right), a=1,2$, depend on which type of boundaries we are considering. Below we display all cases.

Case (I, I):

$$
\zeta_{1}\left(k_{i}, \xi_{+}\right)=\frac{\sin \left(\frac{k_{i}+\eta}{2}+\xi_{+}\right)}{\sin \left(\frac{k_{i}-\eta}{2}-\xi_{+}\right)}, \quad \zeta_{2}\left(k_{i}, \xi_{-}\right)=\frac{\sin \left(\frac{k_{i}+\eta}{2}+\xi_{-}\right)}{\sin \left(\frac{k_{i}-\eta}{2}-\xi_{-}\right)},
$$




$$
\psi_{1}\left(\lambda_{j}, \xi_{+}\right)=\psi_{2}\left(\lambda_{j}, \xi_{-}\right)=1 .
$$

Case (II, II):

$$
\begin{aligned}
& \zeta_{1}\left(k_{i}, \xi_{+}\right)=-\frac{\cos \left(\frac{k_{i}}{2}-\xi_{+}\right)}{\cos \left(\frac{k_{i}}{2}+\xi_{+}\right)}, \quad \zeta_{2}\left(k_{i}, \xi_{-}\right)=-e^{\mathrm{i} k_{i}}, \\
& \psi_{1}\left(\lambda_{j}, \xi_{+}\right)=\frac{\cos \left(\frac{\lambda_{j}-\eta}{2}-\xi_{+}\right) e^{-\mathrm{i} \lambda_{j}}}{\cos \left(\frac{\lambda_{j}+\eta}{2}+\xi_{+}\right)}, \\
& \psi_{2}\left(\lambda_{j}, \xi_{-}\right)=\frac{\cos \left(\frac{\lambda_{j}-\eta}{2}+\xi_{-}\right) e^{\mathrm{i} \lambda_{j}}}{\cos \left(\frac{\lambda_{j}+\eta}{2}-\xi_{+}\right)} .
\end{aligned}
$$

Case (I, II):

$$
\begin{aligned}
& \zeta_{1}\left(k_{i}, \xi_{+}\right)=\frac{\sin \left(\frac{k_{i}+\eta}{2}+\xi_{+}\right)}{\sin \left(\frac{k_{i}-\eta}{2}-\xi_{+}\right)}, \quad \zeta_{2}\left(k_{i}, \xi_{-}\right)=-e^{\mathrm{i} k_{i}}, \\
& \psi_{1}\left(\lambda_{j}, \xi_{+}\right)=1, \quad \psi_{2}\left(\lambda_{j}, \xi_{-}\right)=\frac{\cos \left(\frac{\lambda_{j}-\eta}{2}+\xi_{-}\right) e^{\mathrm{i} \lambda_{j}}}{\cos \left(\frac{\lambda_{j}+\eta}{2}-\xi_{+}\right)} .
\end{aligned}
$$

Case (II, I):

$$
\begin{aligned}
& \zeta_{1}\left(k_{i}, \xi_{+}\right)=-\frac{\cos \left(\frac{k_{i}}{2}-\xi_{+}\right)}{\cos \left(\frac{k_{i}}{2}+\xi_{+}\right)}, \quad \zeta_{2}\left(k_{i}, \xi_{-}\right)=\frac{\sin \left(\frac{k_{i}+\eta}{2}+\xi_{-}\right)}{\sin \left(\frac{k_{i}-\eta}{2}-\xi_{-}\right)}, \\
& \psi_{1}\left(\lambda_{j}, \xi_{+}\right)=\frac{\cos \left(\frac{\lambda_{j}-\eta}{2}-\xi_{+}\right) e^{-\mathrm{i} \lambda_{j}}}{\cos \left(\frac{\lambda_{j}+\eta}{2}+\xi_{+}\right)}, \quad \psi_{2}\left(\lambda_{j}, \xi_{-}\right)=1 .
\end{aligned}
$$

Above $M$ is the number of electrons with spin down and $n$ is the total number of the electrons. Considering in addition the relations (2.23) and (2.24) one can find the energy spectrum for all cases.

Case (I, I):

$$
E=-\frac{\cosh \beta}{c_{1}}-\frac{\cosh \beta}{c_{2}}+4 \sum_{i=1}^{n} \cos k_{i} .
$$

Case (II, II):

$$
E=\exp (\beta)\left(c_{1}+c_{2}\right)+4 \sum_{i=1}^{n} \cos k_{i} .
$$

Case (I, II):

$$
E=\exp (\beta) c_{1}-\frac{\cosh \beta}{c_{2}}+4 \sum_{i=1}^{n} \cos k_{i} .
$$

Case (II, I):

$$
E=-\frac{\cosh \beta}{c_{1}}+\exp (\beta) c_{2}+4 \sum_{i=1}^{n} \cos k_{i} .
$$




\section{Conclusion and discussion}

In summary, we have analysed in detail the open boundary conditions for the Bariev chain with special boundary conditions. Two classes of boundary $K_{+}$-matrices have been obtained by solving the RE. It has been found that these two classes of solutions of the RE lead to four kinds of integrable boundary fields for the charge and spin degrees of freedom separately. Through the nesting procedure, we have diagonalized exactly the two level transfer matrices with four kinds of possible boundary fields. The eigenvalues of the transfer matrices, the energy spectrum and Bethe ansatz equations have been derived for all cases. The boundary fields indeed contribute nontrivially to the ground-state properties as well as the low-lying spectrum. The functions $\zeta_{a}\left(k_{i}, \xi_{ \pm}\right)$and $\psi_{a}\left(\lambda_{j}, \xi_{ \pm}\right)$will contribute nontrivial phase-shift factors to the density of the charge rapidity and spin rapidity. We notice that the Bethe equations (4.95) and (4.96) would be reduced to the purely doubling of the ones for the 1D Bariev model with periodic BC $[25,26]$ if the boundary parameters $\xi_{ \pm} \rightarrow \infty$. These boundary parameters $\xi_{ \pm}$, denoting the impurity strength too, might change the band filling, the boundary surface energy, the mesoscopic effects as well. The results obtained provide us with a basis to investigate the surface critical properties, and correlation functions $[16,32,33]$ for the resulting model.

\section{Acknowledgements}

X.W.G., A.F. and I.R. thank CNPq (Conselho Nacional de Desenvolvimento Científico e Tecnológico) for financial support. J.L. thanks the Australian Research Council for support. H.Q.Z. acknowledges the support from the NNSF of China.

\section{Appendix A. The Boltzman weights and the boundary $K_{\Sigma}$-matrices}

Before practicing our ansatz for fixing the boundary $K_{ \pm}(u)$-matrix, we first display the Boltzmann weights of the $R$-matrix (2.6)

$$
\begin{aligned}
& \rho_{2}=\frac{\sqrt{1+h^{2} u^{2}} \sqrt{1+h^{2} v^{2}}}{1+u v h^{2}} \rho_{1}, \quad \rho_{3}=\frac{(u-v) h}{1+u v h^{2}} \rho_{1}, \\
& \rho_{4}=\frac{\sqrt{1+h^{2} u^{2}} \sqrt{1+h^{2} v^{2}} \sqrt{1+u^{2}} \sqrt{1+v^{2}}}{(1+u v)\left(1+u v h^{2}\right)} \rho_{1}, \\
& \rho_{5}=\frac{h \sqrt{1+h^{2} v^{2}} \sqrt{1+u^{2}}(u-v)}{(1+u v)\left(1+u v h^{2}\right)} \rho_{1}, \\
& \rho_{6}=\frac{\sqrt{1+h^{2} v^{2}} \sqrt{1+u^{2}}(u-v)}{(1+u v)\left(1+u v h^{2}\right)} \rho_{1}, \quad \rho_{7}=\left[1+\frac{h^{2}(u-v)^{2}}{(1+u v)\left(1+u v h^{2}\right)}\right] \rho_{1}, \\
& \rho_{8}=\frac{\sqrt{1+u^{2}} \sqrt{1+v^{2}}}{1+u v} \rho_{1}, \quad \rho_{9}=\frac{(u-v)\left(u-h^{2} v\right)}{(1+u v)\left(1+u v h^{2}\right)} \rho_{1},
\end{aligned}
$$




$$
\begin{aligned}
& \rho_{10}=\left[1+\frac{(u-v)^{2}}{(1+u v)\left(1+u v h^{2}\right)}\right] \rho_{1}, \quad \rho_{11}=\frac{(u-v) h}{1+u v} \rho_{1}, \\
& \rho_{12}=\frac{h \sqrt{1+h^{2} u^{2}} \sqrt{1+v^{2}}(u-v)}{(1+u v)\left(1+u v h^{2}\right)} \rho_{1}, \quad \rho_{13}=\frac{\sqrt{1+h^{2} u^{2}} \sqrt{1+v^{2}}(u-v)}{(1+u v)\left(1+u v h^{2}\right)} \rho_{1}, \\
& \rho_{14}=\frac{(u-v)\left(h^{2} u-v\right)}{(1+u v)\left(1+u v h^{2}\right)} \rho_{1}, \quad \rho_{15}=\frac{h(u-v)^{2}}{(1+u v)\left(1+u v h^{2}\right)} \rho_{1} .
\end{aligned}
$$

As mentioned in Section 2, due to the absence of the isomorphism between $K_{ \pm}$-matrices, we have to solve the RE (2.16), (2.17) separately to fix them. Substituting $K_{-}(u)(2.18)$ into the RE (2.16), we find, at first glance, that the RE (2.16) involve two variables $u$ and $v$ which make the functional equations involving $K a_{-}(u), a=1, \ldots, 4$, much more complicated. However, if we observe the structures of the $R$-matrix and the RE (2.16), we may pick up some simpler functional equations of the RE which allow us to separate $K a_{-}(u), K a_{-}(v)$ into the following forms

$$
\begin{aligned}
& \frac{\mathrm{K} 1_{-}(v)}{\mathrm{K} 2_{-}(v)}=\frac{\rho_{2}(u, v) \rho_{3}(v,-u) \mathrm{K} 1_{-}(u)+\rho_{3}(u, v) \rho_{2}(v,-u) \mathrm{K} 2_{-}(u)}{\rho_{2}(u,-v) \rho_{3}(-v,-u) \mathrm{K} 1_{-}(u)+\rho_{3}(u,-v) \rho_{2}(-v,-u) \mathrm{K} 2_{-}(u)}, \\
& \frac{\mathrm{K} 1_{-}(v)}{\mathrm{K} 3_{-}(v)}=\frac{\rho_{2}(u, v) \rho_{3}(v,-u) \mathrm{K} 1_{-}(u)+\rho_{3}(u, v) \rho_{2}(v,-u) \mathrm{K} 2_{-}(u)}{\rho_{2}(u,-v) \rho_{3}(-v,-u) \mathrm{K} 1_{-}(u)+\rho_{3}(u,-v) \rho_{2}(-v,-u) \mathrm{K} 2_{-}(u)}, \\
& \frac{\mathrm{K} 3_{-}(v)}{\mathrm{K} 4_{-}(v)}=\frac{\rho_{11}(u,-v) \rho_{8}(-v,-u) \mathrm{K} 3_{-}(u)+\rho_{8}(u,-v) \rho_{11}(-v,-u) \mathrm{K} 4_{-}(u)}{\rho_{11}(u, v) \rho_{8}(v,-u) \mathrm{K} 3_{-}(u)+\rho_{8}(u, v) \rho_{2}(v,-u) \mathrm{K} 4_{-}(u)} .
\end{aligned}
$$

Substituting the Boltzmann weights of the $R$-matrix into above equations, and analyzing the consistent conditions of these equations, we can easily conclude an ansatz

$$
\begin{aligned}
& \mathrm{K} 1_{-}(u)=\left(c_{1}+c_{2} u\right)\left(c_{3}+c_{4} u\right)\left(c_{5}+c_{6} u\right), \\
& \mathrm{K} 2_{-}(u)=\left(c_{1}-c_{2} u\right)\left(c_{3}+c_{4} u\right)\left(c_{5}+c_{6} u\right), \\
& \mathrm{K} 3_{-}(u)=\left(c_{1}+c_{2} u\right)\left(c_{3}-c_{4} u\right)\left(c_{5}+c_{6} u\right), \\
& \mathrm{K} 4_{-}(u)=\left(c_{1}+c_{2} u\right)\left(c_{3}-c_{4} u\right)\left(c_{5}-c_{6} u\right)
\end{aligned}
$$

with minimal coefficients $c_{i}$ to be determined. Running the RE again with this ansatz (A.1)-(A.4), it is easily found that only one coefficient is free. Thus the two classes of boundary $K_{-}$-matrices can be immediately chosen as the forms (2.19) and (2.20).

Similarly, substituting $K_{+}$-matrix (2.18) into the RE (2.17) we have

$$
\begin{aligned}
& \frac{\mathrm{K} 1_{+}(v)}{\mathrm{K} 2_{+}(v)}=\frac{\rho_{2}(v, u) \bar{\rho}_{16}(v,-u) \mathrm{K} 1_{+}(u)+\rho_{3}(v, u) \bar{\rho}_{3}(v,-u) \mathrm{K} 2_{+}(u)}{\rho_{3}(-u,-v) \bar{\rho}_{2}(u,-v) \mathrm{K} 1_{+}(u)+\rho_{2}(-u,-v) \bar{\rho}_{16}(u,-v) \mathrm{K} 2_{+}(u)}, \\
& \frac{\mathrm{K} 1_{+}(v)}{\mathrm{K} 3_{+}(v)}=\frac{\rho_{2}(v, u) \bar{\rho}_{16}(v,-u) \mathrm{K} 1_{+}(u)+\rho_{3}(v, u) \bar{\rho}_{2}(v,-u) \mathrm{K} 3_{+}(u)}{\rho_{3}(-u,-v) \bar{\rho}_{3}(u,-v) \mathrm{K} 1_{+}(u)+\rho_{2}(-u,-v) \bar{\rho}_{16}(u,-v) \mathrm{K} 3_{+}(u)}, \\
& \frac{\mathrm{K} 3_{+}(v)}{\mathrm{K} 4_{+}(v)}=\frac{\rho_{8}(-u,-v) \bar{\rho}_{15}(u,-v) \mathrm{K} 3_{+}(u)+\rho_{11}(-u,-v) \bar{\rho}_{7}(u,-v) \mathrm{K} 4_{+}(u)}{\rho_{11}(v, u) \bar{\rho}_{9}(v,-u) \mathrm{K} 3_{+}(u)+\rho_{8}(v, u) \bar{\rho}_{15}(v,-u) \mathrm{K} 4_{+}(u)},
\end{aligned}
$$

here we first have to determine $\bar{\rho}$ 's from the relation (2.9) and (2.10), i.e.,

$$
\bar{\rho}_{2}(x, y)=\frac{\left(1+h^{2} x y\right)^{2}(1+x y) \sqrt{1+h^{2} y^{2}} \sqrt{1+h^{2} x^{2}}}{(x-y)^{2}\left(h^{2} x-y\right)\left(h^{2} y-x\right)},
$$




$$
\begin{aligned}
& \bar{\rho}_{3}(x, y)=\frac{\left(1+h^{2} x y\right)^{2}(1+x y) \sqrt{1+h^{2} y^{2}} \sqrt{1+h^{2} x^{2}}}{(x-y)^{2}\left(h^{2} x-y\right)\left(h^{2} y-x\right) h^{2}}, \\
& \bar{\rho}_{7}(x, y)=\frac{\left(1+h^{2} x y\right)^{2}(1+x y)^{2} \sqrt{1+y^{2}} \sqrt{1+x^{2}}}{(x-y)^{2}\left(h^{2} x-y\right)\left(h^{2} y-x\right)}, \\
& \bar{\rho}_{9}(x, y)=\frac{\left(1+h^{2} x y\right)^{2}(1+x y)^{2} h^{2} \sqrt{1+y^{2}} \sqrt{1+x^{2}}}{(x-y)^{2}\left(h^{2} x-y\right)\left(h^{2} y-x\right)}, \\
& \bar{\rho}_{15}(x, y)=\frac{\left(1+h^{2} x y\right)(1+x y)^{2}}{(x-y)^{2}\left(x h^{2}-y\right)} \\
& \bar{\rho}_{16}(x, y)=\frac{\left(1+h^{2} x y\right)^{2}(1+x y)}{(x-y)^{2}\left(y h^{2}-x\right) h} .
\end{aligned}
$$

With the help of the new Boltzmann weights, the consistency of above equations indeed can be able to lead an ansatz

$$
\begin{aligned}
& \mathrm{K} 1_{+}(u)=\left(c_{1}+c_{2} u\right)\left(c_{3}+c_{4} u h^{2}\right)\left(c_{5}+c_{6} u\right), \\
& \mathrm{K} 2_{+}(u)=\left(c_{2} u-h^{2} c_{1}\right)\left(c_{4} u h^{2}+c_{3}\right)\left(c_{5}+c_{6} u\right), \\
& \mathrm{K} 3_{+}(u)=\left(c_{1}+c_{2} u\right)\left(c_{4} u-c_{3}\right)\left(c_{5}+c_{6} u\right), \\
& \mathrm{K} 4_{+}(u)=\left(c_{1}+c_{2} u\right)\left(c_{4} u-c_{3}\right)\left(c_{6} u-c_{5} h^{2}\right) .
\end{aligned}
$$

Running second RE (2.17) again, the solutions (2.21) and (2.22) would be fixed definitely.

\section{Appendix B. Useful commutation relations}

For factorizing the transfer matrix (2.15) acting on the pseudovacuum state, we need the following commutation relations

$$
\begin{aligned}
C_{1}(u) \bar{B}_{1}(u)= & \frac{\rho_{2}(u,-u)}{\rho_{1}(u,-u)}\left[\bar{B}(u) B(u)-A_{11}(u) \bar{A}_{11}(u)\right], \\
\bar{C}_{1}(u) B_{1}(u)= & \frac{\rho_{2}(u,-u)}{\rho_{1}(u,-u)}\left[B(u) \bar{B}(u)-\bar{A}_{11}(u) A_{11}(u)\right], \\
C_{2}(u) \bar{B}_{2}(u)= & \frac{\rho_{2}(u,-u)}{\rho_{1}(u,-u)}\left[\bar{B}(u) B(u)-A_{22}(u) \bar{A}_{22}(u)\right], \\
\bar{C}_{2}(u) B_{2}(u)= & \frac{\rho_{2}(u,-u)}{\rho_{1}(u,-u)}\left[B(u) \bar{B}(u)-\bar{A}_{22}(u) A_{22}(u)\right], \\
C_{3}(u) \bar{F}(\lambda)= & \frac{\rho_{4}(u,-u)}{\rho_{1}(u,-u)}[\bar{B}(u) B(u)-D(u) \bar{D}(u)] \\
& -\frac{\rho_{2}(u,-u)}{\rho_{1}(u,-u)}\left[C_{4}(u) \bar{E}_{1}(u)+C_{5}(u) \bar{E}_{2}(u)\right], \\
C_{3}(u) \bar{F}(u)= & \frac{\rho_{4}(u,-u)}{\rho_{2}(u,-u)} \bar{C}_{1}(u) B_{1}(u)+\frac{\rho_{8}(u,-u)}{\rho_{2}(u,-u)}\left[\bar{A}_{11}(u) A_{11}(u)-D(u) \bar{D}(u)\right] \\
& -\frac{\rho_{1}(u,-u)}{\rho_{2}(u,-u)} C_{4}(u) \bar{E}_{1}(u)-\frac{\rho_{7}(u,-u)}{\rho_{2}(u,-u)} C_{5}(u) \bar{E}_{2}(u),
\end{aligned}
$$




$$
\begin{aligned}
C_{3}(u) \bar{F}(u)= & \frac{\rho_{4}(u,-u)}{\rho_{2}(u,-u)} \bar{C}_{2}(u) B_{2}(u)+\frac{\rho_{8}(u,-u)}{\rho_{2}(u,-u)}\left[\bar{A}_{22}(u) A_{22}(u)-D(u) \bar{D}(u)\right] \\
& -\frac{\rho_{10}(u,-u)}{\rho_{2}(u,-u)} C_{4}(u) \bar{E}_{1}(u)-\frac{\rho_{1}(u,-u)}{\rho_{2}(u,-u)} C_{5}(u) \bar{E}_{2}(u),
\end{aligned}
$$

which can be driven directly from (3.33). In addition, one also can show the relations $C_{i}(\lambda) \bar{B}_{a}(\lambda)=0$ for $i \neq j, i=1,2,3$, and $a=1,2$. Using these commutation relations and after lengthy algebra, one may manage to present the factorized forms of (3.35) and (3.39).

For checking the eigenvalue problem of two-particle excitation, the following commutation equations are needed

$$
\begin{aligned}
& \rho_{1}(u, v) \rho_{14}(v,-u) \tilde{F}(u) \tilde{B}(v) \\
& =\rho_{14}(u,-v) \rho_{4}(-v,-u) \tilde{F}(v) \tilde{B}(u) \\
& +\rho_{12}(-v,-u)\left[\rho_{3}(u,-v) \tilde{B}_{2}(v) \tilde{B}_{1}(u)+\rho_{12}(u,-v) \tilde{F}_{2}(v) \tilde{A}_{11}(u)\right] \\
& +\rho_{13}(-v,-u)\left[\rho_{3}(u,-v) \tilde{B}_{1}(v) \tilde{B}_{2}(u)+\rho_{13}(u,-v) \tilde{F}_{2}(v) \tilde{A}_{22}(u)\right] \\
& +\rho_{14}(-v,-u)\left[\rho_{1}(u,-v) \tilde{B}(v) \tilde{F}(u)+\rho_{2}(u,-v) \tilde{B}_{1}(v) \tilde{E}_{1}(u)\right. \\
& \left.+\rho_{2}(u,-v) \tilde{B}_{2}(v) \tilde{E}_{2}(u)+\rho_{4}(u,-v) \tilde{F}(v) \tilde{D}(u)\right], \\
& \rho_{9}(u, v) \rho_{14}(v,-u) \tilde{D}(u) \tilde{F}(v) \\
& +\rho_{5}(u, v)\left[\rho_{12}(v,-u) \tilde{A}_{11}(u) \tilde{F}(v)+\rho_{11}(v,-u) \tilde{E}_{1}(u) \tilde{E}_{2}(v)\right] \\
& +\rho_{6}(u, v)\left[\rho_{13}(v,-u) \tilde{A}_{22}(u) \tilde{F}(v)+\rho_{11}(v,-u) \tilde{E}_{2}(u) \tilde{E}_{1}(v)\right] \\
& +\rho_{4}(u, v)\left[\rho_{4}(v,-u) \tilde{B}(u) \tilde{F}(v)+\rho_{8}(v,-u) \tilde{B}_{1}(u) \tilde{E}_{1}(v)\right. \\
& \left.+\rho_{8}(v,-u) \tilde{B}_{2}(u) \tilde{E}_{2}(v)+\rho_{1}(v,-u) \tilde{F}(u) \tilde{D}(v)\right] \\
& =\rho_{4}(u,-v) \rho_{1}(-v,-u) \tilde{B}(v) \tilde{F}(u)+\rho_{1}(u,-v) \rho_{1}(-v,-u) \tilde{F}(v) \tilde{D}(u) \\
& +\rho_{8}(u,-v)\left[\rho_{1}(-v,-u) \tilde{B}_{1}(v) \tilde{E}_{1}(u)+\rho_{1}(-v,-u) \tilde{B}_{2}(v) \tilde{E}_{2}(u)\right], \\
& \rho_{3}(u, v)\left[\rho_{3}(v,-u) \tilde{A}_{11}(u) \tilde{F}(v)+\rho_{13}(v,-u) \tilde{E}_{1}(u) \tilde{E}_{2}(v)\right] \\
& +\rho_{2}(u, v)\left[\rho_{2}(v,-u) \tilde{B}(u) \tilde{F}(v)+\rho_{1}(v,-u) \tilde{B}_{1}(u) \tilde{E}_{1}(v)\right. \\
& \left.+\rho_{10}(v,-u) \tilde{B}_{2}(u) \tilde{E}_{2}(v)+\rho_{8}(v,-u) \tilde{F}(u) \tilde{D}(v)\right] \\
& =\rho_{11}(-v,-u)\left[\rho_{13}(u,-v) \tilde{B}_{2}(v) \tilde{B}_{1}(u)+\rho_{11}(u,-v) \tilde{F}(v) \tilde{A}_{11}(u)\right] \\
& +\rho_{8}(-v,-u)\left[\rho_{2}(u,-v) \tilde{B}(v) \tilde{F}(u)+\rho_{1}(u,-v) \tilde{B}_{1}(v) \tilde{E}_{1}(u)\right. \\
& \left.+\rho_{10}(u,-v) \tilde{B}_{2}(v) \tilde{E}_{2}(u)+\rho_{8}(u,-v) \tilde{F}(v) \tilde{D}(u)\right], \\
& \rho_{3}(u, v)\left[\rho_{3}(v,-u) \tilde{A}_{22}(u) \tilde{F}(v)+\rho_{12}(v,-u) \tilde{E}_{2}(u) \tilde{E}_{1}(v)\right] \\
& +\rho_{2}(u, v)\left[\rho_{2}(v,-u) \tilde{B}(u) \tilde{F}(v)+\rho_{7}(v,-u) \tilde{B}_{1}(u) \tilde{E}_{1}(v)\right. \\
& \left.+\rho_{1}(v,-u) \tilde{B}_{2}(u) \tilde{E}_{2}(v)+\rho_{8}(v,-u) \tilde{F}(u) \tilde{D}(v)\right] \\
& =\rho_{11}(-v,-u)\left[\rho_{13}(u,-v) \tilde{B}_{2}(v) \tilde{B}_{1}(u)+\rho_{11}(u,-v) \tilde{F}(v) \tilde{A}_{22}(u)\right] \\
& +\rho_{8}(-v,-u)\left[\rho_{2}(u,-v) \tilde{B}(v) \tilde{F}(u)+\rho_{7}(u,-v) \tilde{B}_{1}(v) \tilde{E}_{1}(u)\right. \\
& \left.+\rho_{1}(u,-v) \tilde{B}_{2}(v) \tilde{E}_{2}(u)+\rho_{8}(u,-v) \tilde{F}(v) \tilde{D}(u)\right],
\end{aligned}
$$




$$
\begin{aligned}
& \rho_{3}(u, v)\left[\rho_{3}(v,-u) \tilde{A}_{12}(u) \tilde{F}(v)+\rho_{12}(v,-u) \tilde{E}_{1}(u) \tilde{E}_{1}(v)\right] \\
& \quad+\rho_{2}(u, v) \rho_{15}(v,-u) \tilde{B}_{2}(u) \tilde{E}_{1}(v) \\
& =\rho_{11}(-v,-u)\left[\rho_{12}(u,-v) \tilde{B}_{1}(v) \tilde{B}_{2}(u)+\rho_{11}(u,-v) \tilde{F}(v) \tilde{A}_{12}(u)\right] \\
& \quad+\rho_{15}(u,-v) \rho_{8}(-v,-u) \tilde{B}_{2}(v) \tilde{E}_{1}(u), \\
& \rho_{3}(u, v)\left[\rho_{3}(v,-u) \tilde{A}_{21}(u) \tilde{F}(v)+\rho_{13}(v,-u) \tilde{E}_{2}(u) \tilde{E}_{2}(v)\right] \\
& \quad+\rho_{2}(u, v) \rho_{15}(v,-u) \tilde{B}_{1}(u) \tilde{E}_{2}(v) \\
& =\rho_{11}(-v,-u)\left[\rho_{12}(u,-v) \tilde{B}_{1}(v) \tilde{B}_{1}(u)+\rho_{11}(u,-v) \tilde{F}(v) \tilde{A}_{21}(u)\right] \\
& \quad+\rho_{15}(u,-v) \rho_{8}(-v,-u) \tilde{B}_{1}(v) \tilde{E}_{2}(u) .
\end{aligned}
$$

\section{References}

[1] E.H. Lieb, F.Y. Wu, Phys. Rev. Lett. 20 (1968) 1445.

[2] F.H.L. Essler, V.E. Korepin, Exactly Solvable Models of Strongly Correlated Electrons, World Scientific, Singapore, 1994.

[3] P.W. Anderson, Science 235 (1987) 1196;

F.C. Zhang, T.M. Rice, Phys. Rev. B 37 (1988) 3759.

[4] F.H.L. Essler, V.E. Korepin, Phys. Rev. B 46 (1992) 9147;

A. Foerster, M. Karowski, Phys. Rev. B 46 (1992) 9234;

A. Foerster, M. Karowski, Nucl. Phys. B 396 (1993) 611.

[5] H.Q. Zhou, Phys. Rev. B 54 (1996) 41;

H. Asakawa, M. Suzuki, J. Phys. A: Math. Gen. 29 (1996) 225;

X.-W. Guan, M.-S. Wang, S.-D. Yang, Nucl. Phys. B 485 (1997) 685;

X.-W. Guan, M.-S. Wang, S.-D. Yang, J. Phys. A: Math. Gen. 30 (1997) 4161;

M. Shiroishi, M. Wadati, J. Phys. Soc. Jpn. 66 (1997) 2288;

M. Shiroishi, M. Wadati, J. Phys. Soc. Jpn. 66 (1997) 1

[6] F.H.L. Essler, J. Phys. A 29 (1996) 6183;

G. Bedurftig, F.H.L. Essler, H. Frahm, Phys. Rev. Lett. 78 (1997) 1397;

G. Bedurftig, F.H.L. Essler, H. Frahm, Nucl. Phys. B 489 (1997) 697.

[7] K. Hibberd, I. Roditi, J. Links, A. Foerster, Mod. Phys. Lett. A 15 (2000) 133;

H.-Q. Zhou, X.-Y. Ge, J. Links, M.D. Gould, Nucl. Phys. B 546 (1999) 779;

H. Fan, M. Wadati, X.-M. Wang, Phys. Rev. B 61 (2000) 3450.

[8] H.-Q. Zhou, X.-Y. Ge, M.D. Gould, J. Phys. A: Math. Gen. 32 (1999) L137;

A. Foerster, K.E. Hibberd, J. Links, I. Roditi, Phys. Lett. A 271 (2000) 198;

M.J. Martins, X.-W. Guan, Nucl. Phys. B 562 (1999) 433.

[9] L. Mezincescu, R.I. Nepomechie, J. Phys. A: Math. Gen. 24 (1991) L17;

L. Mezincescu, R.I. Nepomechie, Int. J. Mod. Phys. A 7 (1991) 5231;

L. Mezincescu, R.I. Nepomechie, Int. J. Mod. Phys. A 7 (1992) 5657;

R.I. Nepomechie, J. Phys. A: Math. Gen. 33 (2000) L21.

[10] A. Foerster, M. Karowski, Nucl. Phys. B 408 (1993) 512;

J.R. Links, M.D. Gould, Int. J. Mod. Phys. B 10 (1996) 3461;

A.J. Bracken, X.Y. Ge, Y.Z. Zhang, H.Q. Zhou, Nucl. Phys. B 516 (1998) 588.

[11] H.J. de Vega, A. González-Ruiz, J. Phys. A: Math. Gen. 26 (1993) L519;

H.J. de Vega, A. González-Ruiz, Mod. Phys. Lett. A 9 (1994) 2207.

[12] A. González-Ruiz, Nucl. Phys. B 424 (1994) 468.

[13] H. Fan, B.Y. Hou, K.J. Shi, Z.X. Yang, Nucl. Phys. B 478 (1996) 723; 
G.-L. Li, R.-H. Yue, B.-Y. Hou, Nested Bethe ansatz for Perk-Schutz model with open boundary conditions, preprint, 2000;

A. Lima-Santos, Nucl. Phys. B 558 (1999) 637.

[14] E.K. Sklyanin, J. Phys. A: Math. Gen. 21 (1988) 2375.

[15] L.D. Faddeev, in: 1984 Les Houches;

J.B. Zuber, R. Stora (Eds.), Recent Advances in Field Theory and Statistical Mechanics, NorthHolland, Amsterdam, 1982;

P.P. Kulish, E.K. Sklyanin, in: Lecture Notes in Physics, Vol. 151, Springer, Berlin, 1982, p. 61.

[16] V.E. Korepin, N.M. Bogoliubov, A.G. Izergin, Quantum Inverse Scattering Method and Correlation Functions, Cambridge Univ. Press, Cambridge, 1993.

[17] G. Bedürftig, H. Frahm, J. Phys. A: Math. Gen. 32 (1999) 4585;

G. Bedürftig, H. Frahm, Physica E 4 (1999) 246;

H. Asakawa, M. Suzuki, Physica A 236 (1997) 376;

H. Asakawa, Physica A 256 (1998) 229.

[18] F.C. Alcaraz, M.N. Barber, M.T. Batcheler, R.J. Baxter, G.R.W. Quispel, J. Phys. A: Math. Gen. 20 (1987) 6397.

[19] S. Skorik, A. Kapustin, J. Phys. A 29 (1996) 1629;

S. Skorik, H. Saleur, J. Phys. A 28 (1995) 6605.

[20] R.Z. Bariev, J. Phys. A: Math. Gen. 24 (1991) L919.

[21] R.Z. Bariev, A. Klümper, A. Schadschneider, J. Zittartz, J. Phys. A: Math. Gen. 26 (1993) 4663.

[22] H.-Q. Zhou, Phys. Lett. A 221 (1996) 104;

H.-Q. Zhou, J. Phys. A: Math. Gen. 29 (1996) 5509.

[23] M. Shiroishi, M. Wadati, J. Phys. A: Math. Gen. 30 (1997) 1115.

[24] B.S. Shastry, Phys. Rev. Lett. 56 (1986) 1529;

B.S. Shastry, Phys. Rev. Lett. 56 (1986) 2453;

B.S. Shastry, J. Stat. Phys. 50 (1998) 57.

[25] M.J. Martins, P.B. Ramos, J. Phys. A: Math. Gen. 30 (1997) L465.

[26] H.-Q. Zhou, J. Phys. A: Math. Gen. 30 (1997) L423.

[27] H.-Q. Zhou, Phys. Rev. B 53 (1996) 5098;

H.-Q. Zhou, Phys. Lett. A 230 (1997) 45.

[28] A. Yacoby, R. Schuster, M. Meiblum, Phys. Rev. B 53 (1996) 9583;

V.T. Petrashov, V.N. Antonov, P. Delsing, T. Claeson, Phys. Rev. Lett. 74 (1995) 5268.

[29] M.J. Martins, X.-W. Guan, Nucl. Phys. B 583 (2000) 721.

[30] M.J. Martins, P.B. Ramos, Nucl. Phys. B 522 (1998) 413.

[31] X.-W. Guan, J. Phys. A: Math. Gen. 33 (2000) 5391.

[32] A. Klümper, R.Z. Bariev, Nucl. Phys. B 458 (1995) 625;

Y. Fujii, M. Wadati, Chaos Soliton Fract. 11 (2000) 565.

[33] T. Kojima, Finite XXZ critical chain with double boundaries, nlin.SI/0008014. 